\title{
The Semiclassical Propagator for Spin Coherent States
}

\author{
MICHAEL STONE \\ University of Illinois, Department of Physics \\ 1110 W. Green St. \\ Urbana, IL 61801 USA \\ KEE-SU PARK \\ University of Illinois, Department of Physics \\ 1110 W. Green St. \\ Urbana, IL 61801 USA \\ ANUPAM GARG \\ Northwestern University, Department of Physics and Astronomy \\ Evanston, IL 60208 USA
}

\begin{abstract}
We use a continuous-time path integral to obtain the semiclassical propagator for minimal-spread spin coherent states. We pay particular attention to the "extra phase" discovered by Solari and Kochetov, and show that this correction is related to an anomaly in the fluctuation determinant. We show that, once this extra factor is included, the semiclassical propagator has the correct short time behaviour to $O\left(T^{2}\right)$, and demonstrate its consistency under dissection of the path.

PACS numbers: $31.15 . \mathrm{Kb}, 75.45 .+\mathrm{j}$
\end{abstract}

Typeset using REVTEX 


\section{INTRODUCTION}

Coherent-state path integrals for spin were introduced by Klauder [1], and by Kuratsuji and Suzuki [2]. Related phase space path integrals were introduced by Jevicki and Papanicolaou [3], and by Nielsen and Röhrlich [4]. For a review see [5.,6]. These path integrals have attracted attention in connection with geometric quantization [0], and for providing examples hinting at possible infinite-dimensional extensions of the Duistermat-Heckman theorem [8] on conditions for the exactness of the stationary phase approximation [9,10]. Perhaps their most significant practical applications, however, have been in computations of spin tunnelling in the semiclassical limit. Here the spin path-integral formalism gives a good qualitative description of the tunneling process [1] 13], including the simplest and most vivid picture of the topological quenching of spin tunneling [14] that has recently been seen in the magnetic molecule $\mathrm{Fe}_{8}$ [15]. When we require precise quantitative results, however,

the spin coherent-state path integral runs into problems: A straight forward application of instanton methods to compute the tunnel splitting [16, [17] yields answers that are incorrect beyond the leading exponential order [18]. A full derivation of the splitting, including the correct prefactor, has only recently been provided by Belinicher, Providencia and Providencia [19]. These authors showed that the continuum limit of the discrete path integral is rather delicate, and in their computation the simplicity of the instanton method is lost. These difficulties have lead to the spin path integral acquiring a reputation for being unreliable - or, even worse, being meaningful only in its discrete-time form [20]. Many workers in the field have sought alternatives to path integrals such as discrete WKB methods [21 23].

This paper is intended to effect a rehabilitation of the continuous-time spin coherentstate path integral. We advertise and explain the origin of a previously discovered, but largely unknown, correction to the naïve form of the semiclassical propagator. This "extra phase" was obtained by Solari [24] as a result of a careful evaluation of the discrete path form of the path integral. It also appears, as a product of a manipulation, apparently carried out for convenience, in a paper by Kochetov [25]. We derive it here by pointing out 
that the functional determinant resulting from the fluctuation integral about the classical path possesses an anomaly. Regulating the determinant in a manner consistent with the underlying causal structure leads to the extra contribution.

The structure of the paper is as follows: In section two we review spin coherent states built on highest- or lowest-weight spin- $j$ states. We focus primarily on their holomorphic properties. In section three we review the properties of the classical action that appears in the path integral for spin, stressing the importance of boundary terms in avoiding the over-determination problem. In section four we compute the gaussian integral over small fluctuations about the classical path, and obtain the extra-phase correction. In section five we verify that, once the extra contribution is taken into account, the semiclassical propagator has the correct short time behaviour. This verification is immediate at first order in $T$, but the agreement between our expression and the exact result at $O\left(T^{2}\right)$ provides a significant test of the correctness of our result. In section six we check the consistency of the expression for the propagator under the dissection of the path. We find that our semiclassical propagator does not pass this test unless we repartition terms between the exponent and the prefactor. This forces us to regard the large parameter in the semiclassical expansion as being $j+1 / 2$, rather than $j$. As a byproduct, this observation resolves the mystery of the divergent normalization factor that appears in most treatments of the semiclassical propagator. Finally, in section seven, we compute the semiclassical propagator for the hamiltonian $\hat{H}=\nu J_{z}^{2}$. We confirm that our expression obtains the correct leading and next to leading terms in the large- $j$ expansion.

\section{SPIN COHERENT STATES}

We define a family of spin coherent states [26] by

$$
|z\rangle=\exp \left(z \hat{J}_{+}\right)|j,-j\rangle
$$

These states are not normalized, but have the advantage of being holomorphic in the pa-

rameter $z$. Consequently, matrix elements such as $\left\langle z^{\prime}|\hat{O}| z\right\rangle$ will be holomorphic functions of 
the variable $z$, and anti-holomorphic functions of the variable $z^{\prime}$.

The inner product of two of these states is

$$
\left\langle z^{\prime} \mid z\right\rangle=\left(1+\bar{z}^{\prime} z\right)^{2 j}
$$

and the left eigenstates $\langle j, m|$ of $\hat{J}^{2}$ and $\hat{J}_{3}$ have coherent-state wavefunctions

$$
\psi_{m}^{(1)}(z) \equiv\langle j, m \mid z\rangle=\sqrt{\frac{2 j !}{(j-m) !(j+m) !}} z^{j+m} .
$$

This means that a general element of the spin- $j$ Hilbert space may be represented by a polynomial in $z$ of degree $n \leq 2 j$.

As with any family of generalized coherent states derived from a unitary irreducible representation of a compact group, Shur's lemma provides us with an overcompleteness relation. In the present case this reads

$$
\mathbf{1}=\frac{2 j+1}{\pi} \int \frac{d^{2} z}{(1+\bar{z} z)^{2 j+2}}|z\rangle\langle z|
$$

Here $2 j+1$ appears because it is the dimension of the representation. The symbol $d^{2} z$ is shorthand for $d x d y$, and the factor $1 /(1+\bar{z} z)^{2}$ combines with this to make the invariant measure on the coset $S U(2) / U(1)$. This coset is, of course, the two-sphere, $S^{2}$, equipped with stereographic coordinates. The south pole, corresponding to spin down, is at $z=0$, while the north pole, spin up, is at $z=\infty$ - the one-point compactification of the complex plane. The remaining factor in the measure, $1 /(1+\bar{z} z)^{2 j}$, serves to normalize the states.

The wavefunctions $\psi_{m}^{(1)}(z)$ are singular at the north pole, $z=\infty$. Indeed there is no actual state $|\infty\rangle$ because the phase of this putative limiting state would depend on the direction from which we approach the point at infinity. We may, however, define a second family of states

$$
|z\rangle_{2}=\exp \left(z \hat{J}_{-}\right)|j, j\rangle
$$

and form the wavefunctions

$$
\psi_{m}^{(2)}(z)=\langle j, m \mid z\rangle_{2} .
$$


These states and wavefunctions are well defined in the vicinity of the north pole, but singular near the south pole.

To find the relation between $\psi^{(2)}(z)$ and $\psi^{(1)}(z)$ we note that the matrix identity

$$
\left[\begin{array}{ll}
1 & z \\
0 & 1
\end{array}\right]\left[\begin{array}{cc}
0 & 1 \\
-1 & 0
\end{array}\right]=\left[\begin{array}{cc}
1 & 0 \\
z^{-1} & 1
\end{array}\right]\left[\begin{array}{cc}
-z & 0 \\
0 & -z^{-1}
\end{array}\right]\left[\begin{array}{cc}
1 & -z^{-1} \\
0 & 1
\end{array}\right],
$$

coupled with the faithfulness of the spin- $\frac{1}{2}$ representation of $S U(2)$, implies the relation

$$
\exp \left(z \hat{J}_{+}\right) \hat{w}=\exp \left(z^{-1} \hat{J}_{-}\right)(-z)^{2 \hat{J}_{3}} \exp \left(-z^{-1} \hat{J}_{+}\right)
$$

where $\hat{w}=\exp \left(i \pi \hat{J}_{2}\right)$ is the generator of the Weyl group of $S U(2)$. We also note that

$$
\hat{w}|j, j\rangle=(-1)^{2 j}|j,-j\rangle, \quad \hat{w}|j,-j\rangle=|j, j\rangle .
$$

Thus,

$$
\begin{aligned}
\psi_{m}^{(1)}(z) & =\left\langle j, m\left|e^{z \hat{J}_{+}}\right| j,-j\right\rangle \\
& =(-1)^{2 j}\left\langle j, m\left|e^{z \hat{J}_{+}} \hat{w}\right| j, j\right\rangle \\
& =(-1)^{2 j}\left\langle j, m\left|e^{z^{-1} \hat{J}_{-}}(-z)^{2 \hat{J}_{3}} e^{-z^{-1} \hat{J}_{+}}\right| j, j\right\rangle \\
& =(-1)^{2 j}(-z)^{2 j}\left\langle j, m\left|e^{z^{-1} \hat{J}_{-}}\right| j, j\right\rangle \\
& =z^{2 j} \psi_{m}^{(2)}\left(z^{-1}\right) .
\end{aligned}
$$

The coherent-state wavefunctions $\psi_{m}^{(1)}$ and $\psi_{m}^{(2)}$ may therefore be regarded as composing a single global section, $\psi_{m}$, of a holomorphic line bundle with transition function $z^{2 j}$ relating its components $\psi_{m}^{(1)}(z)$, and $\psi_{m}^{(2)}(\zeta \equiv 1 / z)$ in the two coordinate patches. It is the requirement that the transition function and its inverse be holomorphic and single valued in the overlap of the coordinate patches that forces $2 j$ to be an integer. In the sequel, all coherent states, unless otherwise specified, will drawn from the first family, $|z\rangle$.

The above construction is an example of the Borel-Weil realization of representations of compact groups as sections of holomorphic bundles [27. It serves as the paradigm for the more general theory of geometric quantization [7,28]. Because global analyticity is characteristic of the minimal-spread coherent states built on highest- (or lowest-) weight 
states, and also serves (via the transition function) to specify the Hilbert space, it is a property that should be maintained order-by-order in any approximation scheme.

For physical interpretations we must normalize the coherent states. This we do by multiplying them by

$$
N(\bar{z}, z)=(1+\bar{z} z)^{-j}
$$

For example,

$$
N^{2}\left\langle z\left|\hat{J}_{3}\right| z\right\rangle=j \frac{\bar{z} z-1}{\bar{z} z+1}, \quad \text { and } \quad N^{2}\left\langle z\left|\hat{J}_{+}\right| z\right\rangle=\frac{2 j \bar{z}}{\bar{z} z+1}
$$

If we recall the connection between stereographic and spherical polar coordinates,

$$
z=e^{-i \phi} \cot \frac{\theta}{2}
$$

we see that

$$
j \frac{\bar{z} z-1}{\bar{z} z+1}=j \cos \theta, \quad \text { and } \quad \frac{2 j z}{\bar{z} z+1}=j e^{-i \phi} \sin \theta
$$

We also note that

$$
\begin{aligned}
N^{2}\left\langle z\left|\hat{J}_{3}^{2}\right| z\right\rangle & =j\left(j-\frac{1}{2}\right)\left(\frac{\bar{z} z-1}{\bar{z} z+1}\right)^{2}+\frac{1}{2} j \\
& =j\left(j-\frac{1}{2}\right) \cos ^{2} \theta+\frac{j}{2} .
\end{aligned}
$$

Similarly 29]

$$
\begin{aligned}
& N^{2}\left\langle z\left|\hat{J}_{1}^{2}\right| z\right\rangle=j\left(j-\frac{1}{2}\right) \sin ^{2} \theta \cos ^{2} \phi+\frac{j}{2} \\
& N^{2}\left\langle z\left|\hat{J}_{2}^{2}\right| z\right\rangle=j\left(j-\frac{1}{2}\right) \sin ^{2} \theta \sin ^{2} \phi+\frac{j}{2}
\end{aligned}
$$

Thus $N^{2}\left\langle z\left|\hat{\mathbf{J}}^{2}\right| z\right\rangle=j(j+1)$, as it should.

The normalized wavefunctions $N(\bar{z}, z) \psi_{m}^{(1)}(z)$ have their maximum amplitude on the lines of latitude

$$
|z|^{2}=\left|z_{m}\right|^{2}=\frac{j+m}{j-m}
$$


corresponding to the polar angle $\theta_{m}=\cos ^{-1} m / j$. Note that

$$
N^{2}\left\langle z_{m}\left|\hat{J}_{3}\right| z_{m}\right\rangle=j \frac{\left|z_{m}\right|^{2}-1}{\left|z_{m}\right|^{2}+1}=m \text {. }
$$

The variance, in terms of $m$, is given by

$$
\left(N^{2}\left\langle\hat{J}_{3}^{2}\right\rangle-N^{4}\left\langle\hat{J}_{3}\right\rangle^{2}\right)=\frac{1}{2} j\left(1-\left(\frac{\bar{z} z-1}{\bar{z} z+1}\right)^{2}\right)=\frac{1}{2} j\left(1-\cos ^{2} \theta\right) .
$$

Since $m \sim j \cos \theta$, the normalized wavefunctions have zonal spread $\Delta \theta \sim 1 / \sqrt{j}$. As $j$ becomes large the quantum spin becomes more localized, and more classical.

\section{SPIN ACTION}

We wish to find a semiclassical approximation for the propagator

$$
K\left(\bar{\zeta}_{f}, \zeta_{i}, T\right)=\left\langle\zeta_{f}\left|e^{-i \hat{H} T}\right| \zeta_{i}\right\rangle
$$

in the form

$$
K_{\mathrm{scl}}\left(\bar{\zeta}_{f}, \zeta_{i}, T\right)=K_{\text {reduced }} \cdot \exp \left\{S_{\mathrm{cl}}\left(\bar{\zeta}_{f}, \zeta_{i}, T\right)\right\}
$$

Here $S_{\mathrm{cl}}$ is the action for a classical path going from the point $z=\zeta_{i}$ to the point $z=\zeta_{f}$ in time $T$. The action functional is expected to be that appearing in the path integral representation of the exact propagator. The amplitude $K_{\text {reduced }}$, the pre-exponential factor, is then given by a gaussian approximation to the integral over deviations from the classical trajectory. Such a semiclassical approximation should be accurate when $j$ is large.

If a continuous-time path integral is "derived" by inserting $N$ intermediate overcompleteness relations into (3.1) and taking a formal limit $N \rightarrow \infty$, then we find 25]

$$
K\left(\bar{\zeta}_{f}, \zeta_{i}, T\right)=\int_{\zeta_{i}}^{\bar{\zeta}_{f}} d \mu(\bar{z}, z) \exp \{S(\bar{z}(t), z(t))\}
$$

where the path measure $d \mu$ is

$$
d \mu(\bar{z}(t), z(t))=\lim _{N \rightarrow \infty} \prod_{n=1}^{N} \frac{2 j+1}{\pi} \frac{d^{2} z_{n}}{\left(1+\bar{z}_{n} z_{n}\right)^{2 j+2}},
$$


and the action $S(\bar{z}(t), z(t))$ is given by

$$
S(\bar{z}(t), z(t))=j\left\{\ln \left(1+\bar{\zeta}_{f} z(T)\right)+\ln \left(1+\bar{z}(0) \zeta_{i}\right)\right\}+\int_{0}^{T}\left\{j \frac{\dot{\bar{z}} z-\bar{z} \dot{z}}{1+\bar{z} z}-i H(\bar{z}, z)\right\} d t
$$

Here the classical hamiltonian, $H(\bar{z}, z)$, is related to the quantum $\hat{H}$ by

$$
H(\bar{z}, z)=\langle z|\hat{H}| z\rangle /\langle z \mid z\rangle
$$

The paths $z(t), \bar{z}(t)$ obey the boundary conditions $z(0)=\zeta_{i}, \bar{z}(T)=\bar{\zeta}_{f}$, but $\bar{z}(0), z(T)$, being actually $\bar{z}(0+\epsilon)$ and $z(T-\epsilon)$, are unconstrained, and are to be integrated over [25].

When we regard $S$ as the phase-space action for a classical system [31], the explicit boundary terms, which appear naturally in the discretized path integral, serve to ensure that both the first-order Hamilton equations and their boundary conditions are compatible with the action principle. To see this, make a general variation in the trajectory, including variations in the endpoints. We find that

$$
\begin{aligned}
\delta S= & \frac{2 j z(T)}{1+\bar{\zeta}_{f} z(T)} \delta \bar{\zeta}_{f}+\frac{2 j \bar{z}(0)}{1+\bar{z}(0) \zeta_{i}} \delta \zeta_{i} \\
& +\int_{0}^{T}\left\{\delta z(t)\left(\frac{2 j \dot{\bar{z}}}{(1+\bar{z} z)^{2}}-i \frac{\partial H}{\partial z}\right)+\delta \bar{z}(t)\left(-\frac{2 j \dot{z}}{(1+\bar{z} z)^{2}}-i \frac{\partial H}{\partial \bar{z}}\right)\right\} d t
\end{aligned}
$$

There are no boundary contributions proportional to $\delta \bar{z}(0)$ or $\delta z(T)$ because of a cancellation of such terms arising from an integration by parts against those arising from the variation of the explicit boundary terms. Equating the variation of the action to zero therefore requires the classical path to obey the Hamilton equations

$$
\dot{\bar{z}}=i \frac{(1+\bar{z} z)^{2}}{2 j} \frac{\partial H}{\partial z}, \quad \dot{z}=-i \frac{(1+\bar{z} z)^{2}}{2 j} \frac{\partial H}{\partial \bar{z}}
$$

together with boundary conditions that fix $z(0)=\zeta_{i}$, and $\bar{z}(T)=\bar{\zeta}_{f}$.

The quantities $\bar{z}(0)$ and $z(T)$ are not fixed by the boundary conditions, but can be found by solving the equations of motion. If we know the action for the classical path, they can also be read off from the Hamilton-Jacobi equations that follow from (3.7), viz:

$$
\frac{\partial S_{\mathrm{cl}}}{\partial \bar{\zeta}_{f}}=\frac{2 j z(T)}{1+\bar{\zeta}_{f} z(T)}, \quad \frac{\partial S_{\mathrm{cl}}}{\partial \zeta_{i}}=\frac{2 j \bar{z}(0)}{1+\bar{z}(0) \zeta_{i}}
$$


In general $\bar{z}(0)$ will not be the complex conjugate of $z(0) \equiv \zeta_{i}$, nor will $z(T)$ be the complex conjugate of $\bar{z}(T) \equiv \bar{\zeta}_{f}$. This means that if we write $z$ as $x+i y$ and $\bar{z}=x-i y$, then, except in special cases, $x$ and $y$ are not real numbers.

The Hamilton-Jacobi relations also tell us that

$$
\frac{\partial S_{\mathrm{cl}}}{\partial \bar{\zeta}_{i}}=\frac{\partial S_{\mathrm{cl}}}{\partial \zeta_{f}}=0
$$

showing that $S_{\mathrm{cl}}$ is a holomorphic function of $\zeta_{i}$, and an anti-holomorphic function of $\zeta_{f}$. These analyticity properties of $S_{\mathrm{cl}}$ coincide with those of $K$. This is reasonable since $\exp S_{\mathrm{cl}}$ is the leading approximation to $K$, and we would expect analyticity to preserved term-by-term in the large $j$ expansion. Finally

$$
\frac{\partial S_{\mathrm{cl}}}{\partial T}=-i H\left(\bar{\zeta}_{f}, z(T)\right)
$$

The leading semiclassical approximation is exact when the quantum hamiltonian $\hat{H}$ is an element of the Lie algebra of $S U(2)$. For example, if $\hat{H}=\omega \hat{J}_{3}$, then

$$
H(\bar{z}, z)=N^{2}\langle z|\hat{H}| z\rangle=\omega j \frac{\bar{z} z-1}{\bar{z} z+1}
$$

and

$$
\frac{\partial H}{\partial z}=\frac{2 j \omega \bar{z}}{(1+\bar{z} z)^{2}}, \quad \frac{\partial H}{\partial \bar{z}}=\frac{2 j \omega z}{(1+\bar{z} z)^{2}}
$$

The equations of motion are therefore

$$
\dot{\bar{z}}=i \omega \bar{z}, \quad \dot{z}=-i \omega z
$$

The solutions obeying the appropriate boundary conditions are

$$
z(t)=e^{-i \omega t} \zeta_{i}, \quad \bar{z}(t)=e^{i \omega(t-T)} \bar{\zeta}_{f}
$$

So

$$
z(T)=e^{-i \omega T} \zeta_{i}, \quad \bar{z}(0)=e^{-i \omega T} \bar{\zeta}_{f}
$$

It will only be in exceptional circumstances that $z(T)=\left(\bar{\zeta}_{f}\right)^{*}$ or $\bar{z}(0)=\left(\zeta_{i}\right)^{*}$. 
Inserting the solutions (3.15) into the action we find

$$
\begin{aligned}
S_{\mathrm{cl}}\left(\bar{\zeta}_{f}, \zeta_{i}, T\right) & =j\left\{\ln \left(1+\bar{\zeta}_{f} \zeta_{i} e^{-i \omega T}\right)+\ln \left(1+\bar{\zeta}_{f} \zeta_{i} e^{-i \omega T}\right)\right\}+\int_{0}^{T}\left\{i j \omega \frac{2 \bar{z} z}{1+\bar{z} z}-i j \omega \frac{\bar{z} z-1}{z z+1}\right\} d t \\
& =2 j \ln \left(1+\bar{\zeta}_{f} \zeta_{i} e^{-i \omega T}\right)+i j \omega T .
\end{aligned}
$$

This is to be compared with the exact propagator

$$
K=\left\langle\zeta_{f}\left|e^{-i \hat{H} T}\right| \zeta_{i}\right\rangle=e^{i \omega j T}\left(1+e^{-i \omega T} \bar{\zeta}_{f} \zeta_{i}\right)^{2 j}=\exp S_{\mathrm{cl}}
$$

When the hamiltonian is a more general element of the enveloping algebra (i.e. a polynomial in the generators) there will be corrections to this simple result.

\section{FLUCTUATION DETERMINANT}

The prefactor in the semiclassical propagator comes from integration over gaussian fluctuations about the classical trajectory. To evaluate these, we consider the second variation of the classical action, holding $z(0)=\zeta_{i}$ and $\bar{z}(T)=\bar{\zeta}_{f}$ fixed. We will write

$$
S=S_{\mathrm{cl}}+\delta S+\frac{1}{2} \delta^{2} S+\cdots
$$

where

$$
\delta^{2} S=-i \int_{0}^{T} \frac{2 j}{(1+\bar{z} z)^{2}}\left(\begin{array}{ll}
\delta \bar{z} & \delta z
\end{array}\right)\left[\begin{array}{cc}
-i \partial_{t}+A & B \\
\bar{B} & i \partial_{t}+A
\end{array}\right]\left(\begin{array}{l}
\delta z \\
\delta \bar{z}
\end{array}\right) d t
$$

Here

$$
\begin{aligned}
A & =\frac{1}{2}\left(\frac{\partial}{\partial \bar{z}} \frac{(1+\bar{z} z)^{2}}{2 j} \frac{\partial H}{\partial z}+\frac{\partial}{\partial z} \frac{(1+\bar{z} z)^{2}}{2 j} \frac{\partial H}{\partial \bar{z}}\right) \\
B & =\frac{\partial}{\partial \bar{z}} \frac{(1+\bar{z} z)^{2}}{2 j} \frac{\partial H}{\partial \bar{z}} \\
\bar{B} & =\frac{\partial}{\partial z} \frac{(1+\bar{z} z)^{2}}{2 j} \frac{\partial H}{\partial z}
\end{aligned}
$$

When $z(t), \bar{z}(t)$ are the classical path, then $\delta S=0$.

On making a change of variables 


$$
\begin{aligned}
& \delta z=(1+\bar{z} z) \eta, \\
& \delta \bar{z}=(1+\bar{z} z) \bar{\eta},
\end{aligned}
$$

we see that we have to compute the quadratic path integral

$$
K_{\text {reduced }} \propto \int d[\eta] d[\bar{\eta}] \exp \left\{-2 i j \int_{0}^{T} \frac{1}{2}\left(\begin{array}{ll}
\bar{\eta} & \eta
\end{array}\right)\left[\begin{array}{cc}
-i \partial_{t}+A & B \\
\bar{B} & i \partial_{t}+A
\end{array}\right]\left(\begin{array}{l}
\eta \\
\bar{\eta}
\end{array}\right) d t\right\} .
$$

This path integral is proportional to $\operatorname{Det}^{-\frac{1}{2}} \mathcal{D}$, where the matrix differential operator

$$
\mathcal{D}=\left[\begin{array}{cc}
-i \partial_{t}+A & B \\
\bar{B} & i \partial_{t}+A
\end{array}\right]=-i \sigma_{3} \partial_{t}+M
$$

is subject to the boundary conditions $\eta(0)=0$ and $\bar{\eta}(T)=0$. (We will use the symbol "Det" for functional determinants and "det" to denote the determinant of a finite matrix. Similarly "Tr" and "tr".)

There are several subtleties involved in calculating Det $\mathcal{D}$. The most obvious is that the boundary conditions imposed on $\mathcal{D}$ are not in the class that make it self adjoint. Although $\mathcal{D}$ and $\mathcal{D}^{\dagger}$ are formally the same differential operator, self-adjointness requires, in addition, that their domains of definition coincide [30]. It is not hard to see that the only boundary condition on $\mathcal{D}$ that leads to an identical boundary condition for $\mathcal{D}^{\dagger}$ is $\eta(0)=e^{i \theta_{0}} \bar{\eta}(0)$ and $\eta(T)=e^{i \theta_{T}} \bar{\eta}(T)$ for some real angles $\theta_{0}, \theta_{T}$. Indeed if $B=\bar{B}=0$, then $\mathcal{D}$ with our boundary conditions has no eigenfunctions - never mind a complete set. The determinant cannot be expressed as an infinite product of eigenvalues, therefore. Diagonalizability is not, however, a fundamental requirement for defining a determinant. There exists a well-defined Green function $G=\mathcal{D}^{-1}$, and we should be able to obtain the determinant by varying the parameters and using the identity $\delta \ln \operatorname{Det} \mathcal{D}=\operatorname{Tr}\left\{\mathcal{D}^{-1} \delta \mathcal{D}\right\}$, which holds even if $\mathcal{D}$ is not diagonalizable.

A potential pitfall in this approach is that the variation $\delta \ln \operatorname{Det} \mathcal{D}$ is given by

$$
\delta \ln \operatorname{Det} \mathcal{D}=\operatorname{Tr}\left\{\mathcal{D}^{-1} \delta \mathcal{D}\right\}=\int_{0}^{T} \operatorname{tr}\{G(t, t) \delta M\} d t
$$

but the Green function $G\left(t, t^{\prime}\right)$ is discontinuous at $t=t^{\prime}$. We might have a different expression for the variation depending on whether we choose to evaluate $G(t, t)$ as $G(t, t+\epsilon)$ or as 
$G(t, t-\epsilon)$. The jump in $G$ is, however, proportional to $\sigma_{3}$, and $\operatorname{tr}\left\{\sigma_{3} \delta M\right\} \equiv 0$, so we have reason to hope that there is no actual ambiguity.

If we agree to interpret $G(t, t)$ as $\frac{1}{2}(G(t, t+\epsilon)+G(t, t-\epsilon))$, then the formal calculation is straight forward [32], and we merely summarize the results:

We begin by defining the matrix

$$
\Phi(t)=\left(\begin{array}{cc}
\eta_{T}(t) & \eta_{0}(t) \\
\bar{\eta}_{T}(t) & \bar{\eta}_{0}(t)
\end{array}\right) .
$$

Here the column vector $\left(\eta_{0}(t), \bar{\eta}_{0}(t)\right)^{T}$ is a solution of $\mathcal{D} \Psi=0$ obeying the boundary condition $\eta_{0}(0)=0, \bar{\eta}_{0}(0)=1$, and $\left(\eta_{T}(t), \bar{\eta}_{T}(t)\right)^{T}$ is a solution with $\eta_{T}(T)=1, \bar{\eta}_{T}(T)=0$. The determinant of $\Phi(t)$ is an analogue of the wronskian and is independent of $t$. We find that $\operatorname{Det} \mathcal{D}=C \operatorname{det} \Phi$, where $C$ is some constant independent of $H$.

Since $\operatorname{det} \Phi$ is time independent, we may conveniently evaluate it at $t=T$, where

$$
C^{-1} \operatorname{Det} \mathcal{D}=\left|\begin{array}{cc}
1 & \eta_{0}(T) \\
0 & \bar{\eta}_{0}(T)
\end{array}\right|=\bar{\eta}_{0}(T)
$$

or at $t=0$, where

$$
C^{-1} \operatorname{Det} \mathcal{D}=\left|\begin{array}{cc}
\eta_{T}(0) & 0 \\
\bar{\eta}_{T}(0) & 1
\end{array}\right|=\eta_{T}(0)
$$

By relaxing the conditions that $\eta(T)=\bar{\eta}(0)=1$, we may interpret these results in terms of the variation of the endpoints of the classical trajectory as we vary the initial points. That is

$$
C^{-1} \operatorname{Det} \mathcal{D}=\left(\frac{\partial \bar{\eta}(0)}{\partial \bar{\eta}(T)}\right)^{-1}=\left(\frac{\partial \eta(T)}{\partial \eta(0)}\right)^{-1},
$$

or, in terms of the original variables,

$$
C^{-1} \operatorname{Det} \mathcal{D}=\frac{1+\bar{z}(0) \zeta_{i}}{1+\bar{\zeta}_{f} z(T)}\left(\frac{\partial \bar{z}(0)}{\partial \bar{\zeta}_{f}}\right)^{-1}=\frac{1+\bar{\zeta}_{f} z(T)}{1+\bar{z}(0) \zeta_{i}}\left(\frac{\partial z(T)}{\partial \zeta_{i}}\right)^{-1}
$$

The equivalence of these two expressions for the determinant is not immediately obvious, but from the Hamilton-Jacobi relations

$$
\frac{\partial S_{\mathrm{cl}}}{\partial \bar{\zeta}_{f}}=\frac{2 j z(T)}{1+\bar{\zeta}_{f} z(T)}, \quad \frac{\partial S_{\mathrm{cl}}}{\partial \zeta_{i}}=\frac{2 j \bar{z}(0)}{1+\bar{z}(0) \zeta_{i}}
$$


and the equality of mixed partials of $S_{\mathrm{cl}}$, we obtain

$$
\frac{\partial^{2} S_{\mathrm{cl}}}{\partial \zeta_{i} \partial \bar{\zeta}_{f}}=\frac{2 j}{\left(1+\bar{\zeta}_{f} z(T)\right)^{2}} \frac{\partial z(T)}{\partial \zeta_{i}}=\frac{2 j}{\left(1+\bar{z}(0) \zeta_{i}\right)^{2}} \frac{\partial \bar{z}(0)}{\partial \bar{\zeta}_{f}}
$$

Both expressions in (4.12) thus reduce to

$$
C \operatorname{Det}^{-1} \mathcal{D}=\frac{\left(1+\bar{\zeta}_{f} z(T)\right)\left(1+\bar{z}(0) \zeta_{i}\right)}{2 j} \frac{\partial^{2} S}{\partial \zeta_{i} \partial \bar{\zeta}_{f}}
$$

Our calculation of the fluctuation determinant suggests, therefore, that

$$
K_{\mathrm{scl}}\left(\bar{\zeta}_{f}, \zeta_{i}, T\right) \stackrel{?}{=}\left(\frac{\left(1+\bar{\zeta}_{f} z(T)\right)\left(1+\bar{z}(0) \zeta_{i}\right)}{2 j} \frac{\partial^{2} S_{\mathrm{cl}}}{\partial \zeta_{i} \partial \bar{\zeta}_{f}}\right)^{\frac{1}{2}} \exp S_{\mathrm{cl}}\left(\bar{\zeta}_{f}, \zeta_{i}, T\right)
$$

(The proportionality constant is fixed by the requirement that this expression reduces to $\left\langle\zeta_{f} \mid \zeta_{i}\right\rangle$ when $\left.T=0\right)$.

As indicated by the "?" over the equals sign, there are problems with this expression, and it is not quite correct.

The first problem is that, despite the optimism expressed above, there is a degree of indeterminacy in the calculation of the functional determinant. To see this, make the substitution

$$
\begin{aligned}
& \eta(t) \rightarrow e^{i \theta(t)} \eta(t), \\
& \bar{\eta}(t) \rightarrow e^{-i \theta(t)} \bar{\eta}(t)
\end{aligned}
$$

in the path integral (4.5). The measure is unchanged, but we replace $\mathcal{D}$ with $\tilde{\mathcal{D}}$, where $\tilde{\mathcal{D}}$ is the matrix operator $\mathcal{D}$ with

$$
\begin{aligned}
& A \rightarrow \tilde{A}=A+\partial_{t} \theta, \\
& B \rightarrow \tilde{B}=e^{-2 i \theta(t)} B, \\
& \bar{B} \rightarrow \tilde{\bar{B}}=e^{2 i \theta(t)} \bar{B} .
\end{aligned}
$$

The value of the path integral must be unaltered by this change of integration variables, but the solution to 


$$
\left[\begin{array}{cc}
-i \partial_{t}+\tilde{A} & \tilde{B} \\
\tilde{\bar{B}} & i \partial_{t}+\tilde{A}
\end{array}\right]\left(\begin{array}{l}
\eta(t) \\
\bar{\eta}(t)
\end{array}\right)=0
$$

with $\eta(0)=0, \bar{\eta}(0)=1$ is now $\left(e^{-i(\theta(t)-\theta(0))} \eta_{0}(t), e^{i(\theta(t)-\theta(0))} \bar{\eta}_{0}(t)\right)^{T}$. The determinant, as we have calculated it, is therefore not invariant, but ends up multiplied by $e^{-i(\theta(T)-\theta(0))}$. Our expression for the functional determinant has an "anomaly" therefore.

The anomaly arises because the argument we made about the harmlessness of the discontinuity in $G$ depends on our defining $G(t, t)$ as $G(t, t \pm \epsilon)$ with the same choice of sign in front of the $\epsilon$ in both entries in the trace. If we examine the discrete version of path integral we see that, on the contrary, one of the entries should be evaluated with a plus, and one with a minus. Our calculation of the determinant assumed that we could interpret $G(t, t)$ as $\frac{1}{2}(G(t, t+\epsilon)+G(t, t-\epsilon))$, so our formula for the determinant is only correct if both terms in $\operatorname{tr}\left\{\sigma_{3} \delta M\right\}$ are separately zero. This will only be the case for operators $\mathcal{D}$ with $A \equiv 0$. Fortunately the discrete path integral does permit the change of variables described above, and we may use this freedom to force the diagonal entries, $\tilde{A}$, to zero before computing the determinant. The correctly regulated functional determinant therefore differs from its naïve value by a multiplicative factor.

Including the correction to the fluctuation determinant, the semiclassical propagator becomes

$$
K_{\mathrm{scl}}\left(\bar{\zeta}_{f}, \zeta_{i}, T\right)=\left(\frac{\left(1+\bar{\zeta}_{f} z(T)\right)\left(1+\bar{z}(0) \zeta_{i}\right)}{2 j} \frac{\partial^{2} S_{\mathrm{cl}}}{\partial \zeta_{i} \partial \bar{\zeta}_{f}}\right)^{\frac{1}{2}} \exp \left\{S_{\mathrm{cl}}\left(\bar{\zeta}_{f}, \zeta_{i}, T\right)+\frac{i}{2} \int_{0}^{T} A(t) d t\right\} .
$$

where

$$
A(\bar{z}, z)=\frac{1}{2}\left(\frac{\partial}{\partial \bar{z}} \frac{(1+\bar{z} z)^{2}}{2 j} \frac{\partial H}{\partial z}+\frac{\partial}{\partial z} \frac{(1+\bar{z} z)^{2}}{2 j} \frac{\partial H}{\partial \bar{z}}\right)
$$

is the coefficient appearing in (4.3)

The manoeuvre of setting $\tilde{A}$ to zero before evaluating the fluctuation determinant appears (although without explanation as to why it was necessary) in the previously cited paper by Kochetov [25] that provided part of the motivation for our present work. Kochetov therefore 
gets the corrected expression (4.20). It seems, however, that the "extra phase" (it is a phase only in the simplest cases), $\frac{i}{2} \int_{0}^{T} A(t) d t$, was first obtained by Solari 24 from a careful evaluation of the discrete determinant. Solari also pointed out the necessity of a similar correction in the harmonic oscillator coherent-state path integral, which has a flat phase space. Kochetov's discovery of the correction seems to have been independent of this earlier work.

Because of the extra phase, (4.20) gives the correct, indeed exact, semiclassical propagator for the case $\hat{H}=\omega \hat{J}_{z}$, and also for any hamiltonian consisting of (possibly time dependent) elements of the Lie algebra of $S U(2)$ [25].

\section{SHORT TIME ACCURACY}

The Solari-Kochetov phase also solves a second problem with (4.16). In contrast to the configuration space propagator, which diverges as $T^{-\frac{1}{2}}$, the coherent-state propagator $K\left(\zeta_{f}, \zeta_{i}, T\right)$ is analytic in $T$ near $T=0$. This is because of the finite spread of the coherentstate wavefunctions. To first order in $T$ we have

$$
\begin{aligned}
K\left(\bar{\zeta}_{f}, \zeta_{i}, T\right) \equiv\left\langle\zeta_{f}\left|e^{-i \hat{H} T}\right| \zeta_{i}\right\rangle & \approx\left\langle\zeta_{f} \mid \zeta_{i}\right\rangle-i T\left\langle\zeta_{f}|\hat{H}| \zeta_{i}\right\rangle \\
& =\left\langle\zeta_{f} \mid \zeta_{i}\right\rangle\left(1-i T H\left(\bar{\zeta}_{f}, \zeta_{i}\right)\right)
\end{aligned}
$$

(In the last equality we have exploited analyticity to observe that the off-diagonal $\left\langle\zeta_{f}|\hat{H}| \zeta_{i}\right\rangle$, is obtained from the diagonal $\langle\zeta|\hat{H}| \zeta\rangle$ by the the simple replacement $\zeta \rightarrow \zeta_{i}, \bar{\zeta} \rightarrow \bar{\zeta}_{f}$.)

Now, from the Hamilton-Jacobi equation

$$
\frac{\partial S_{\mathrm{cl}}}{\partial T}=-i H\left(\bar{\zeta}_{f}, z(T)\right)
$$

we have

$$
S_{\mathrm{cl}}\left(\bar{\zeta}_{f}, \zeta_{i}, T\right)=S_{\mathrm{cl}}\left(\bar{\zeta}_{f}, \zeta_{i}, 0\right)-i T H\left(\bar{\zeta}_{f}, \zeta_{i}\right)+O\left(T^{2}\right)
$$

while 


$$
S_{\mathrm{cl}}\left(\bar{\zeta}_{f}, \zeta_{i}, 0\right)=2 j \ln \left(1+\bar{\zeta}_{f} \zeta_{i}\right)=\ln \left\langle\zeta_{f} \mid \zeta_{i}\right\rangle
$$

Thus, in order to get agreement between (4.20) and (5.1), the fluctuation determinant must make no $O(T)$ contribution to the propagator. A short calculation shows, however, that

$$
\frac{\left(1+\bar{\zeta}_{f} z(T)\right)\left(1+\bar{z}(0) \zeta_{i}\right)}{2 j} \frac{\partial^{2} S_{\mathrm{cl}}}{\partial \zeta_{i} \partial \bar{\zeta}_{f}}=1-i T A\left(\bar{\zeta}_{f}, \zeta_{i}\right)+O\left(T^{2}\right) .
$$

Fortunately this contribution is exactly cancelled by the $O(T)$ contribution from the SolariKochetov extra phase.

We now ask how well does the semiclassical propagator do at next order in the shorttime expansion. In order to provide a systematic grading for the terms, we will regard the hamiltonian $\hat{H}$ as being $O(j)$. The entire action is then homogeneous of degree one in $j$. With this assumption, and by analogy with the usual semiclassical expansion in powers of $\hbar$, we expect that

$$
K\left(\bar{\zeta}_{f}, \zeta_{i}, T\right)=K_{\text {reduced }} \cdot \exp \left\{S_{\mathrm{cl}}\right\} \cdot\left[1+O\left(\frac{1}{j}\right)\right]
$$

where $S_{\mathrm{cl}}$ is $O(j)$, while the prefactor, $K_{\text {reduced }}$, is $O\left(j^{0}\right)$.

At short time the exact coherent-state propagator is certainly of this form. To demonstrate this, expand

$$
\left\langle\zeta_{f}\left|e^{-i \hat{H} T}\right| \zeta_{i}\right\rangle=\left\langle\zeta_{f} \mid \zeta_{i}\right\rangle-i T\left\langle\zeta_{f}|\hat{H}| \zeta_{i}\right\rangle-\frac{T^{2}}{2}\left\langle\zeta_{f}\left|\hat{H}^{2}\right| \zeta_{i}\right\rangle+\cdots
$$

Now $\left\langle\zeta_{f}|\hat{H}| \zeta_{i}\right\rangle=\left\langle\zeta_{f} \mid \zeta_{i}\right\rangle H\left(\bar{\zeta}_{f}, \zeta_{i}\right)$, but some work is needed to evaluate $\left\langle\zeta_{f}\left|\hat{H}^{2}\right| \zeta_{i}\right\rangle$.

Inserting an overcompleteness integral, we have

$$
\begin{aligned}
\left\langle\zeta_{f}\left|\hat{H}^{2}\right| \zeta_{i}\right\rangle & =\frac{2 j+1}{\pi} \int \frac{d^{2} z}{(1+\bar{z} z)^{2 j+2}}\left\langle\zeta_{f}|\hat{H}| z\right\rangle\left\langle z|\hat{H}| \zeta_{i}\right\rangle \\
& =\frac{2 j+1}{\pi} \int \frac{d^{2} z}{(1+\bar{z} z)^{2 j+2}}\left(1+\bar{\zeta}_{f} z\right)^{2 j}\left(1+\bar{z} \zeta_{i}\right)^{2 j} H\left(\bar{\zeta}_{f}, z\right) H\left(\bar{z}, \zeta_{i}\right) .
\end{aligned}
$$

We now perform a steepest descent expansion in the integral over the intermediate states, and obtain the first three terms in its asymptotic expansion in powers of $1 / j$. This computation is greatly simplified by using two shortcuts: First we need calculate only the diagonal matrix 
element $\left\langle\zeta\left|\hat{H}^{2}\right| \zeta\right\rangle$. Given this, we may appeal to analyticity and obtain the general matrix element by setting $\bar{\zeta} \rightarrow \bar{\zeta}_{f}$ and $\zeta \rightarrow \zeta_{i}$. Next we rotate the sphere so as to centre the coordinate system on the point $\zeta$. Thus $\zeta \rightarrow 0$, and the coordinate system is locally geodetic. In these coordinates the saddle point of the $z$ integral is at $\zeta=z=0$, and far fewer terms have to taken into consideration.

To return to the original coordinates, we need to be able to recognize some $S O(3) \simeq$ $S U(2)$ invariant combinations of derivatives and $(1+\bar{z} z)^{2}$ factors.

One easily establishes that, under the Möbius mapping

$$
z \rightarrow z^{\prime}=\frac{a z+b}{c z+d}, \quad \text { where } \quad\left[\begin{array}{ll}
a & b \\
c & d
\end{array}\right] \in S U(2),
$$

we have

$$
\frac{d^{2} z}{(1+\bar{z} z)^{2}}=\frac{d^{2} z^{\prime}}{\left(1+\bar{z}^{\prime} z^{\prime}\right)^{2}}
$$

together with

$$
\begin{aligned}
(1+\bar{z} z)^{2} \frac{\partial f(\bar{z}, z)}{\partial z} \frac{\partial g(\bar{z}, z)}{\partial \bar{z}} & =\left(1+\bar{z}^{\prime} z^{\prime}\right)^{2} \frac{\partial f\left(\bar{z}^{\prime}, z^{\prime}\right)}{\partial z^{\prime}} \frac{\partial g\left(\bar{z}^{\prime}, z^{\prime}\right)}{\partial \bar{z}^{\prime}}, \\
(1+\bar{z} z)^{2} \frac{\partial^{2} f(\bar{z}, z)}{\partial z \partial \bar{z}} & =\left(1+\bar{z}^{\prime} z^{\prime}\right)^{2} \frac{\partial^{2} f\left(\bar{z}, z^{\prime}\right)}{\partial z^{\prime} \partial \bar{z}^{\prime}}
\end{aligned}
$$

and that the combination

$$
Z=\left(\frac{\partial}{\partial z}(1+\bar{z} z)^{2} \frac{\partial}{\partial z} f\right)\left(\frac{\partial}{\partial \bar{z}}(1+\bar{z} z)^{2} \frac{\partial}{\partial \bar{z}} g\right)
$$

is similarly invariant. Thus, when we see the term $\partial_{z z}^{2} f \partial_{z z}^{2} g$ appearing in the expansion about the stationary point $z=0$, we realize that in the integral for the general matrix element (where the saddle point is at $z=\zeta_{i}, \bar{z}=\bar{\zeta}_{f}$ ) we should replace it by (5.12).

Proceeding in this manner we find

$$
\begin{aligned}
\left\langle\zeta_{f}\left|\hat{H}^{2}\right| \zeta_{i}\right\rangle=\left\langle\zeta_{f} \mid \zeta_{i}\right\rangle & \left\{H^{2}\left(\bar{\zeta}_{f}, \zeta_{i}\right)+\frac{\left(1+\bar{\zeta}_{f} \zeta_{i}\right)^{2}}{2 j} \frac{\partial H}{\partial \zeta_{i}} \frac{\partial H}{\partial \bar{\zeta}_{f}}\right. \\
& \left.+\frac{1}{2} \frac{1}{(2 j)^{2}}\left(\frac{\partial}{\partial \bar{\zeta}_{f}}\left(1+\bar{\zeta}_{f} \zeta_{i}\right)^{2} \frac{\partial H}{\partial \bar{\zeta}_{f}}\right)\left(\frac{\partial}{\partial \zeta_{i}}\left(1+\bar{\zeta}_{f} \zeta_{i}\right)^{2} \frac{\partial H}{\partial \zeta_{i}}\right)+O\left(\frac{1}{j}\right)\right\} .
\end{aligned}
$$

The three terms in braces in this expression are of $O\left(j^{2}\right), O(j)$, and of $O\left(j^{0}\right)$ respectively. 
We may now re-exponentiate (5.13) as

$$
\begin{aligned}
\left\langle\zeta_{f}\left|e^{-i \hat{H} T}\right| \zeta_{i}\right\rangle= & \exp \left\{\ln \left\langle\zeta_{f} \mid \zeta_{i}\right\rangle-i T H\left(\bar{\zeta}_{f}, \zeta_{i}\right)-\frac{1}{2} T^{2} \frac{\left(1+\bar{\zeta}_{f} \zeta_{i}\right)^{2}}{2 j} \frac{\partial H}{\partial \zeta_{i}} \frac{\partial H}{\partial \bar{\zeta}_{f}}+\cdots\right\} \\
& \times\left[1-\frac{T^{2}}{4} \cdot \frac{1}{(2 j)^{2}}\left(\frac{\partial}{\partial \zeta_{i}}\left(1+\bar{\zeta}_{f} \zeta_{i}\right)^{2} \frac{\partial}{\partial \zeta_{i}} H\right)\left(\frac{\partial}{\partial \bar{\zeta}_{f}}\left(1+\bar{\zeta}_{f} \zeta_{i}\right)^{2} \frac{\partial}{\partial \bar{\zeta}_{f}} H\right)+\cdots\right]
\end{aligned}
$$

Again using the Hamilton Jacobi equation,

$$
\frac{\partial S_{\mathrm{cl}}}{\partial T}=-i H\left(\bar{\zeta}_{f}, z(T)\right)
$$

and the equation of motion for $z(t)$, we may generate the Taylor series for $S_{\mathrm{cl}}(T)$. We immediately verify the term in the exponential is the classical action to $O\left(T^{2}\right)$ :

$$
S_{\mathrm{cl}}=\ln \left\langle\zeta_{f} \mid \zeta_{i}\right\rangle-i T H\left(\bar{\zeta}_{f}, \zeta_{i}\right)-\frac{1}{2} T^{2} \frac{\left(1+\bar{\zeta}_{f} \zeta_{i}\right)^{2}}{2 j} \frac{\partial H}{\partial \zeta_{i}} \frac{\partial H}{\partial \bar{\zeta}_{f}}+O\left(T^{3}\right) .
$$

The expression in the square brackets in (5.14) must be the prefactor, and is manifestly $O\left(j^{0}\right)$. It is a little tedious to verify that our formula for the pre-exponential factor, including the Solari-Kochetov correction, reduces to exactly this, but it is so. To collapse the terms, it helps to use the identity

$$
\begin{aligned}
& (1+\bar{z} z)^{2} \frac{\partial^{2}}{\partial \bar{z} \partial z}\left((1+\bar{z} z)^{2} \frac{\partial H}{\partial \bar{z}} \frac{\partial H}{\partial z}\right)= \\
& 2(1+\bar{z} z)^{2} \frac{\partial H}{\partial \bar{z}} \frac{\partial H}{\partial z}+\left((1+\bar{z} z)^{2} \frac{\partial^{2} H}{\partial \bar{z} \partial z}\right)^{2}+(1+\bar{z} z)^{2} \frac{\partial H}{\partial \bar{z}} \frac{\partial}{\partial z}\left((1+\bar{z} z)^{2} \frac{\partial^{2} H}{\partial \bar{z} \partial z}\right) \\
& \quad+(1+\bar{z} z)^{2} \frac{\partial H}{\partial z} \frac{\partial}{\partial \bar{z}}\left((1+\bar{z} z)^{2} \frac{\partial^{2} H}{\partial \bar{z} \partial z}\right)+\left(\frac{\partial}{\partial z}(1+\bar{z} z)^{2} \frac{\partial}{\partial z} H\right)\left(\frac{\partial}{\partial \bar{z}}(1+\bar{z} z)^{2} \frac{\partial}{\partial \bar{z}} H\right)
\end{aligned}
$$

which is most easily established by noting that all terms are invariant, and, at $z=0$, both sides reduce to

$$
\left(\frac{\partial^{2}}{\partial \bar{z} \partial z}+2\right) \frac{\partial H}{\partial \bar{z}} \frac{\partial H}{\partial z}
$$

The semiclassical expression, therefore, has errors of at most $O\left(j^{-1}\right)$ at short time. Our expectation is, of course, that it has this degree of accuracy uniformly in $T$. 


\section{CONSISTENCY}

A further test of the correctness of (4.20) is to verify its consistency under dissection of the classical trajectory. The exact propagator must satisfy the sewing condition

$$
K\left(\bar{\zeta}_{f}, \zeta_{i}, t_{1}+t_{2}\right)=\frac{2 j+1}{\pi} \int \frac{d^{2} \xi}{(1+\bar{\xi} \xi)^{2 j+2}} K\left(\bar{\zeta}_{f}, \xi, t_{2}\right) K\left(\bar{\xi}, \zeta_{i}, t_{1}\right)
$$

which follows from the definition of $K$ and the overcompleteness condition (2.4). The semiclassical approximation to $K$ should obey a similar condition, but with the exact integration over the intermediate states replaced by a suitable stationary phase approximation.

Since $K_{\mathrm{scl}} \sim \exp S_{\mathrm{cl}}$, we begin with the relationship between the action for the total path from $\zeta_{i}$ to $\zeta_{f}$, and the actions for the two segments from $\zeta_{i}$ to the intermediate point $\xi$, and

from $\xi$ to $\zeta_{f}$. To eliminate the redundant intermediate-point boundary terms we must define

$$
S\left(\bar{\zeta}_{f}, \zeta_{i}, t_{1}+t_{2}\right)=S\left(\bar{\zeta}_{f}, \xi, t_{2}\right)+S\left(\bar{\xi}, \zeta_{i}, t_{1}\right)-2 j \ln (1+\bar{\xi} \xi)
$$

We will write this compactly as

$$
S_{\mathrm{tot}}=S_{2}+S_{1}-2 j \ln (1+\bar{\xi} \xi)
$$

In writing (6.2) we have tacitly assumed that our chosen starting $\xi$ of the second path segment coincides with the dynamically determined endpoint $z\left(t_{1}\right)$ of the first path segment, and that the dynamically determined starting $\bar{z}\left(t_{1}\right)$ of the second path segment coincides with our chosen $\bar{\xi}$ endpoint of the first path segment. This will not generally be the case but it will be when $\bar{\xi}$, $\xi$ obey the stationary-phase equations

$$
\frac{\partial S_{\mathrm{tot}}}{\partial \xi}=\frac{\partial S_{\mathrm{tot}}}{\partial \bar{\xi}}=0
$$

Taking into account the analyticity properties of $S_{1}$ and $S_{2}$, these are

$$
\begin{aligned}
0 & =\frac{\partial S_{2}\left(\bar{\zeta}_{f}, \xi\right)}{\partial \xi}-\frac{2 j \bar{\xi}}{1+\bar{\xi} \xi} \\
0 & =\frac{\partial S_{1}\left(\bar{\xi}, \zeta_{i}\right)}{\partial \bar{\xi}}-\frac{2 j \xi}{1+\bar{\xi} \xi} .
\end{aligned}
$$


Comparing (6.5) with the Hamilton-Jacobi equations confirms that $\xi_{c}=z\left(t_{1}\right)$ and $\bar{\xi}_{c}=\bar{z}\left(t_{1}\right)$, where $\xi_{c}, \bar{\xi}_{c}$ is the stationary phase point.

To evaluate the integral over small deviations from the classical stationary phase point, we set $\xi=\xi_{c}+\eta, \bar{\xi}=\bar{\xi}_{c}+\bar{\eta}$. We expand

$$
S_{\mathrm{tot}}=\left.S_{\mathrm{tot}}\right|_{\bar{\xi}_{c}, \xi_{c}}-\frac{1}{2} \frac{2 j}{\left(1+\bar{\xi}_{c} \xi_{c}\right)^{2}}(\bar{\eta}, \quad \eta)\left[\begin{array}{cc}
1 & -\alpha \\
-\beta & 1
\end{array}\right]\left(\begin{array}{l}
\eta \\
\bar{\eta}
\end{array}\right),
$$

where

$$
\alpha=\frac{\left(1+\bar{\xi}_{c} \xi_{c}\right)^{2}}{2 j} \frac{\partial^{2} S_{1}}{\partial \bar{\xi}_{c}^{2}}+\xi_{c}^{2}=\frac{1}{2 j}\left(1+\bar{\xi}_{c} \xi_{c}\right) \frac{\partial}{\partial \bar{\xi}_{c}}\left(1+\bar{\xi}_{c} \xi_{c}\right) \frac{\partial S_{1}}{\partial \bar{\xi}_{c}}
$$

and

$$
\beta=\frac{\left(1+\bar{\xi}_{c} \xi_{c}\right)^{2}}{2 j} \frac{\partial^{2} S_{2}}{\partial \xi_{c}^{2}}+\bar{\xi}_{c}^{2}=\frac{1}{2 j}\left(1+\bar{\xi}_{c} \xi_{c}\right) \frac{\partial}{\partial \xi_{c}}\left(1+\bar{\xi}_{c} \xi_{c}\right) \frac{\partial S_{2}}{\partial \xi_{c}} .
$$

(The second equality in these equations uses the stationary phase equations.)

We now put together two semiclassical propagators and perform the gaussian integral over the deviation from the stationary phase point. Using the semiclassical Solari-Kochetov form (4.20) for the propagators on the right-hand side of Eq. (6.1), we get (with $T=t_{1}+t_{2}$ ),

$$
\begin{aligned}
K_{\mathrm{comb}}= & \frac{2 j+1}{\pi} \int \frac{d^{2} \eta}{\left(1+\bar{\xi}_{c} \xi_{c}\right)^{2}} \exp \left\{S_{1}+S_{2}-2 j \ln \left(1+\bar{\xi}_{c} \xi_{c}\right)+\frac{i}{2} \int_{0}^{T} A d t-\frac{1}{2} \delta^{2} S\right\} \\
& \times\left(\frac{\left(1+\bar{\zeta}_{f} z(T)\right)\left(1+\bar{\xi}_{c} \xi_{c}\right)}{2 j} \frac{\partial^{2} S_{2}}{\partial \bar{\zeta}_{f} \partial \xi_{c}} \frac{\left(1+\bar{\xi}_{c} \xi_{c}\right)\left(1+\bar{z}(0) \zeta_{i}\right)}{2 j} \frac{\partial^{2} S_{1}}{\partial \bar{\xi}_{c} \partial \zeta_{i}}\right)^{\frac{1}{2}} .
\end{aligned}
$$

Notice that, as with consistency test of the ordinary Feynman path integral [33], the measure and the prefactors, including the Solari-Kochetov "extra-phase" term, are all being treated as constants. The integration involves only the variation of the classical action

$$
\delta^{2} S=\frac{2 j}{\left(1+\bar{\xi}_{c} \xi_{c}\right)^{2}}(\bar{\eta}, \quad \eta)\left[\begin{array}{cc}
1 & -\alpha \\
-\beta & 1
\end{array}\right]\left(\begin{array}{l}
\eta \\
\bar{\eta}
\end{array}\right)
$$

and yields, along with other factors, the inverse square-root of the determinant

$$
D=\left|\begin{array}{cc}
1 & -\alpha \\
-\beta & 1
\end{array}\right|
$$

We now use the result, established in the appendix, that 


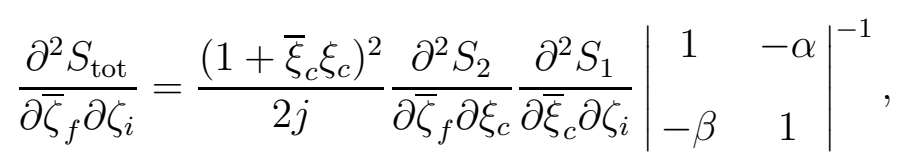

to obtain

$$
K_{\mathrm{comb}}=\left(\frac{2 j+1}{2 j}\right)\left(\frac{\left(1+\bar{\zeta}_{f} z(T)\right)\left(1+\bar{z}(0) \zeta_{i}\right)}{2 j} \frac{\partial^{2} S_{\mathrm{tot}}}{\partial \bar{\zeta}_{f} \partial \zeta_{i}}\right)^{\frac{1}{2}} \exp \left\{S_{\mathrm{tot}}\left(\bar{\zeta}_{f}, \zeta_{i}, T\right)+\frac{i}{2} \int_{0}^{T} A d t\right\} .
$$

The semiclassical approximation therefore reproduces itself except for a niggling factor of $(2 j+1) / 2 j$, which is due to a conflict between the normalization of the measure and the $2 j$ appearing in the exponent.

Although this discrepant factor approaches unity in the large- $j$ limit, it is nonetheless disturbing. Each of the infinitely many gaussian integrations that constitute the semiclassical approximation to the path integral ought to be indistinguishable from our single gaussian integration over the intermediate point $\xi$. We should, therefore, be able to dissect the path into arbitrarily many parts without affecting the final answer. This is not currently so, and, in particular, the limit of large $j$ does not commute with the limit of a large number of intermediate points.

The origin of the discrepancy is not hard to find. In the large- $j$ limit the effective radius of our spherical phase space becomes large, and, near $z=0$, the spin- $j$ reproducing-kernel relation

$$
\frac{2 j+1}{\pi} \int \frac{d^{2} z}{(1+\bar{z} z)^{2}}(1+\bar{z} z)^{-2 j}\left\langle\zeta_{2} \mid z\right\rangle\left\langle z \mid \zeta_{1}\right\rangle=\left\langle\zeta_{2} \mid \zeta_{1}\right\rangle
$$

or more explicitly,

$$
\frac{2 j+1}{\pi} \int \frac{d^{2} z}{(1+\bar{z} z)^{2}}(1+\bar{z} z)^{-2 j}\left(1+\bar{\zeta}_{2} z\right)^{2 j}\left(1+\bar{z} \zeta_{1}\right)^{2 j}=\left(1+\bar{\zeta}_{2} \zeta_{1}\right)^{2 j},
$$

should contract to a suitably scaled version of its flat-phase-space analogue

$$
\int \frac{d^{2} z}{\pi} e^{-\bar{z} z} e^{\bar{\zeta}_{2} z} e^{\bar{z} \zeta_{1}}=e^{\bar{\zeta}_{2} \zeta_{1}}
$$

Because it is a gaussian integral, the leading stationary phase "approximation" to (6.16) is exact. 
If we make the obvious large $j$ estimates

$$
(1+\bar{z} z)^{-2 j} \sim e^{-2 j \bar{z} z}, \quad\left(1+\bar{\zeta}_{2} z\right)^{2 j} \sim e^{2 j \bar{\zeta}_{2} z}, \quad\left(1+\bar{z} \zeta_{1}\right)^{2 j} \sim e^{2 j \bar{z} \zeta_{1}},
$$

while regarding the sphere measure $(1+\bar{z} z)^{-2}$ as a prefactor, we do not get exactly

$$
\frac{2 j}{\pi} \int d^{2} z e^{-2 j \bar{z} z} e^{2 j \bar{\zeta}_{2} z} e^{2 j \bar{z} \zeta_{1}}=e^{2 j \bar{\zeta}_{2} \zeta_{1}}
$$

but instead $(2 j+1) / 2 j$ times this.

If we keep terms higher order in $1 / 2 j$, both those coming from the measure and those from going beyond the quadratic approximation to the exponent, they will of course correct the error. What we really need, however, is a partitioning of the integral on the LHS of (6.15) such that the leading steepest descent approximation will agree with the RHS. This will happen if regard the expansion parameter as $2 j+1$ and not $2 j$. To see this, break up

$$
I=\frac{2 j+1}{\pi} \int \frac{d^{2} z}{(1+\bar{z} z)^{2}}(1+\bar{z} z)^{-2 j}\left(1+\bar{\zeta}_{2} z\right)^{2 j}\left(1+\bar{z} \zeta_{1}\right)^{2 j}
$$

as

$$
I=\frac{2 j+1}{\pi} \int \frac{d^{2} z}{(1+\bar{z} z)^{2}} g^{-1}(\bar{z}, z) e^{(2 j+1) \ln g(\bar{z}, z)}
$$

with

$$
g(\bar{z}, z)=(1+\bar{z} z)^{-1}\left(1+\bar{\zeta}_{2} z\right)\left(1+\bar{z} \zeta_{1}\right) .
$$

The critical point of the function in the exponential is at $\bar{z}=\bar{\zeta}_{2}, z=\zeta_{1}$, and

$$
\begin{gathered}
g\left(\bar{\zeta}_{2}, \zeta_{1}\right)=\left(1+\bar{\zeta}_{2} \zeta_{1}\right), \\
-\left.\frac{\partial^{2} \ln g}{\partial z \partial \bar{z}}\right|_{\bar{z}=\bar{\zeta}_{2}, z=\zeta_{1}}=\frac{1}{\left(1+\bar{\zeta}_{2} \zeta_{1}\right)^{2}} .
\end{gathered}
$$

Thus

$$
\begin{aligned}
I & \sim \frac{2 j+1}{\pi} \frac{1}{\left(1+\bar{\zeta}_{2} \zeta_{1}\right)^{3}}\left(1+\bar{\zeta}_{2} \zeta_{1}\right)^{2 j+1} \int d^{2} z e^{-\frac{2 j+1}{\left(1+\bar{\zeta}_{2} \zeta_{1}\right)^{2}}\left(\bar{z}-\bar{\zeta}_{2}\right)\left(z-\zeta_{1}\right)} \\
& =\frac{2 j+1}{\pi}\left(1+\bar{\zeta}_{2} \zeta_{1}\right)^{2 j-2} \cdot \frac{\pi}{2 j+1}\left(1+\bar{\zeta}_{2} \zeta_{1}\right)^{2} \\
& =\left(1+\bar{\zeta}_{2} \zeta_{1}\right)^{2 j} .
\end{aligned}
$$


The leading term of the asymptotic expansion of $I$ in powers of $1 /(2 j+1)$ is therefore exact.

This observation suggests rewriting the semiclassical approximation to $K$ as

$$
K_{\mathrm{scl}}\left(\bar{\zeta}_{f}, \zeta_{i}, T\right)=\frac{1}{\sqrt{2 j+1}}\left(\frac{\partial^{2} \tilde{S}_{\mathrm{cl}}}{\partial \bar{\zeta}_{f} \partial \zeta_{i}}\right)^{\frac{1}{2}} \exp \left\{\tilde{S}_{\mathrm{cl}}\left(\bar{\zeta}_{f}, \zeta_{i}, T\right)+\frac{i}{2} \int_{0}^{T} Q d t\right\}
$$

where $\tilde{S}_{\mathrm{cl}}=(2 j+1) S_{\mathrm{cl}} /(2 j)$, and

$$
Q=\frac{1}{j}\left(\frac{(1+\bar{z} z)^{2}}{2} \frac{\partial^{2} H}{\partial \bar{z} \partial z}+H(\bar{z}, z)\right)
$$

is the term required to make (6.25) numerically equal to (4.20).

With this repartitioning of terms between the exponent and the prefactor we have exactly the same classical equations of motion, but now

$$
\begin{aligned}
K_{\mathrm{comb}}= & \frac{2 \tilde{j}}{\pi} \int \frac{d^{2} \eta}{\left(1+\bar{\xi}_{c} \xi_{c}\right)^{2}}\left(1+\bar{\xi}_{c} \xi_{c}\right) \exp \left\{\tilde{S}_{1}+\tilde{S}_{2}-(2 \tilde{j}) \ln \left(1+\bar{\xi}_{c} \xi_{c}\right)-\frac{1}{2} \delta^{2} \tilde{S}\right\} \\
& \times \frac{1}{(2 \tilde{j})}\left(\frac{\partial^{2} \tilde{S}_{2}}{\partial \bar{\zeta}_{f} \partial \xi_{c}} \frac{\partial^{2} \tilde{S}_{1}}{\partial \bar{\xi}_{c} \partial \zeta_{i}}\right)^{\frac{1}{2}} \exp \left\{\frac{i}{2} \int_{0}^{T} Q d t\right\},
\end{aligned}
$$

where

$$
\tilde{j}=j+\frac{1}{2}
$$

and

$$
\delta^{2} \tilde{S}=\frac{2 \tilde{j}}{\left(1+\bar{\xi}_{c} \xi_{c}\right)^{2}}(\bar{\eta}, \quad \eta)\left[\begin{array}{cc}
1 & -\tilde{\alpha} \\
-\tilde{\beta} & 1
\end{array}\right]\left(\begin{array}{l}
\eta \\
\bar{\eta}
\end{array}\right) .
$$

The quantities $\tilde{\alpha}$ and $\tilde{\beta}$ are obtained from Eqs. (6.7) and (6.8) by putting tildes on $S_{1}, S_{2}$, and $j$. Note, though, that $\tilde{\alpha}=\alpha$, and $\tilde{\beta}=\beta$. Note also, that we have inserted a factor of $\left(1+\bar{\xi}_{c} \xi_{c}\right)$ in the integral to compensate for the extra factor of $(1+\bar{\xi} \xi)$ that was taken from the measure into the exponential to complete $\tilde{S}_{\text {tot }}$. Thus part of both the measure and the prefactor are varied in determining the stationary phase, and get integrated over, while part is regarded as a constant.

The integration in Eq. (6.27) can be done at once by noting that all equations in Appendix A are unchanged if we put tildes on the actions, $j, \alpha$, and $\beta$ everywhere. In particular, the identity (A9) holds with tildes. We thus obtain 


$$
K_{\mathrm{comb}}=\frac{1}{\sqrt{2 j+1}}\left(\frac{\partial^{2} \tilde{S}_{\mathrm{tot}}}{\partial \bar{\zeta}_{f} \partial \zeta_{i}}\right)^{\frac{1}{2}} \exp \left\{\tilde{S}_{\mathrm{tot}}\left(\bar{\zeta}_{f}, \zeta_{i}, T\right)+\frac{i}{2} \int_{0}^{T} Q d t\right\}
$$

all unwanted factors of $2 j+1$ and $\left(1+\bar{\xi}_{c} \xi_{c}\right)$, having cancelled. Thus, with this form of stationary-phase integration, the propagator reproduces itself exactly.

What this means is that the semiclassical approximation must be tacitly using (6.24) in making each of the many integrations that go into the gaussian approximation to the path integral. Once we realize this, we see that there is no need for the mysterious divergent normalization factor, $\mathcal{N}=\lim _{N \rightarrow \infty}(1+1 / 2 j)^{N}$, that plagues most treatments of the semiclassical spin propagator.

The appearance of $j+1 / 2$ as the large parameter in the fluctuation integral has been remarked on before by Ercolessi et al. [34] and by Funahashi et al. [35]. The former worry that it is inconsistent to include fluctuations of the measure in the gaussian integral without also considering their effect in the saddle point equations. In our case all terms that are being integrated over do appear also in the equations determining the saddle point.

Note that the correction $Q$ vanishes for Larmor precession where $\hat{H}=\omega \hat{J}_{3}$. In this case, as we have seen earlier,

$$
S_{\mathrm{cl}}=2 j \ln \left(1+\bar{\zeta}_{f} \zeta_{i} e^{-i \omega T}\right)+i j \omega T
$$

$\tilde{S}$ is obtained from this by the substitution $j \rightarrow j+\frac{1}{2}$, so

$$
\frac{\partial^{2} \tilde{S}}{\partial \bar{\zeta}_{f} \partial \zeta_{i}}=e^{-i \omega T} \frac{2 j+1}{\left(1+\bar{\zeta}_{f} \zeta_{i} e^{-i \omega T}\right)^{2}}
$$

Thus

$$
\begin{aligned}
\frac{1}{\sqrt{2 j+1}}\left(\frac{\partial^{2} \tilde{S}}{\partial \bar{\zeta}_{f} \partial \zeta_{i}}\right)^{\frac{1}{2}} e^{\tilde{S}\left(\bar{\zeta}_{f}, \zeta_{i}, T\right)} & =e^{-i \omega T / 2}\left(1+\bar{\zeta}_{f} \zeta_{i} e^{-i \omega T}\right)^{-1}\left(1+\bar{\zeta}_{f} \zeta_{i} e^{-i \omega T}\right)^{2 j+1} e^{i \omega\left(j+\frac{1}{2}\right) T} \\
& =e^{i \omega T}\left(1+\bar{\zeta}_{f} \zeta_{i} e^{-i \omega T}\right)^{2 j}
\end{aligned}
$$

which is the exact answer. 


\section{AN EXAMPLE: $\hat{H}=\nu \hat{J}_{3}^{2}$}

As an application of the semiclassical formalism consider $\hat{H}=\nu \hat{J}_{3}^{2}$. This hamiltonian is time reversal invariant, and we might worry that a hidden shift $j \rightarrow j+1 / 2$ would compromise the Kramers degeneracy expected when $j$ is half integral.

The classical hamiltonian corresponding to $\hat{H}=\nu \hat{J}_{3}^{2}$ is

$$
H(\bar{z}, z)=\frac{\left\langle z\left|\nu \hat{J}_{3}^{2}\right| z\right\rangle}{\langle z \mid z\rangle}=\nu\left(j\left(j-\frac{1}{2}\right)\left(\frac{\bar{z} z-1}{\bar{z} z+1}\right)^{2}+\frac{1}{2} j\right) .
$$

This should be compared with the "naïve" classical hamiltonian

$$
H_{\text {naive }}=\nu j^{2}\left(\frac{\bar{z} z-1}{\bar{z} z+1}\right)^{2}
$$

which is what we would get if we simply expressed the classical direction-dependent energy $\nu j^{2} \cos ^{2} \theta$ in terms of the stereographic coordinates on $S^{2}$.

The hamiltonian (7.1) leads to the classical equations of motion

$$
\dot{\bar{z}}=i \omega(\bar{z}, z) \bar{z}, \quad \dot{z}=-i \omega(\bar{z}, z) z
$$

where, with $\mu=\nu j(j-1 / 2)$,

$$
\omega(\bar{z}, z)=\left(\frac{2 \mu}{j}\right)\left(\frac{\bar{z} z-1}{\bar{z} z+1}\right) .
$$

Since these equations imply the time independence of the product $\bar{z} z, \omega$ is itself time independent and the solutions may be written down directly as

$$
z(t)=e^{-i \omega t} \zeta_{i}, \quad \bar{z}(t)=e^{i \omega(t-T)} \bar{\zeta}_{f}
$$

Here $\omega$ is to be determined by the self-consistency condition

$$
\omega=\left(\frac{2 \mu}{j}\right)\left(\frac{e^{-i \omega T} \bar{\zeta}_{f} \zeta_{i}-1}{e^{-i \omega T} \bar{\zeta}_{f} \zeta_{i}+1}\right)
$$

As we will see below, this equation has an infinite family of solutions. Here, we wish to consider how various quantities scale with $j$. By demanding that Eqs. (7.3) continue to be meaningful as $j \rightarrow \infty$, we see that we must have $\mu=O(j), \omega=O(1)$, and $\nu=O(1 / j)$. 
The classical action for the solution $(7.5)$ is

$$
\begin{aligned}
S_{\mathrm{cl}}\left(\bar{\zeta}_{f}, \zeta_{i}, T\right)= & 2 j \ln \left(1+e^{-i \omega T} \bar{\zeta}_{f} \zeta_{i}\right) \\
& +\int_{0}^{T}\left\{j\left(\frac{2 i \omega e^{-i \omega T} \bar{\zeta}_{f} \zeta_{i}}{1+e^{-i \omega T} \bar{\zeta}_{f} \zeta_{i}}\right)-i \mu \omega^{2}\left(\frac{j}{2 \mu}\right)^{2}-\frac{i}{2} j \nu\right\} d t \\
= & 2 j \ln \left(1+e^{-i \omega T} \bar{\zeta}_{f} \zeta_{i}\right)+i T\left\{j \omega+\frac{j^{2}}{4 \mu} \omega^{2}-\frac{1}{2} j \nu\right\} .
\end{aligned}
$$

The apparently cosmetic rewrite in the last line leads to a useful way of looking at the problem. Define

$$
S_{\omega}\left(\bar{\zeta}_{f}, \zeta_{i}, T\right)=2 j \ln \left(1+e^{-i \omega T} \bar{\zeta}_{f} \zeta_{i}\right)+i T\left\{j \omega+\frac{j^{2}}{4 \mu} \omega^{2}\right\}
$$

where we regard $\omega$ as an independent variable. The equation

$$
\frac{\partial S_{\omega}\left(\bar{\zeta}_{f}, \zeta_{i}, T\right)}{\partial \omega}=i T j\left\{-\frac{e^{-i \omega T} \bar{\zeta}_{f} \zeta_{i}-1}{e^{-i \omega T} \bar{\zeta}_{f} \zeta_{i}+1}+\left(\frac{j}{2 \mu}\right) \omega\right\}
$$

then shows that the consistency condition on $\omega$ is equivalent to $\partial S_{\omega} / \partial \omega=0$. We can also use $S_{\omega}\left(\bar{\zeta}_{f}, \zeta_{i}, T\right)$ to express the second variation of $S_{\mathrm{cl}}$ required for the prefactor $A$. By differentiating the Jacobi equation (3.9) we have

$$
\frac{\partial^{2} S_{\mathrm{cl}}\left(\bar{\zeta}_{f}, \zeta_{i}, T\right)}{\partial \bar{\zeta}_{f} \partial \zeta_{i}}=\frac{2 j}{\left(1+\bar{\zeta}_{f} z(T)\right)^{2}} \frac{\partial z(T)}{\partial \zeta_{i}}
$$

and from this we find, with Eq. (7.5), that

$$
\frac{\partial^{2} S_{\mathrm{cl}}\left(\bar{\zeta}_{f}, \zeta_{i}, T\right)}{\partial \bar{\zeta}_{f} \partial \zeta_{i}}=\frac{2 j}{\left(1+\bar{\zeta}_{f} z(T)\right)^{2}}\left\{e^{-i \omega T}+e^{-i \omega T} \zeta_{i}\left(-i T \frac{\partial \omega}{\partial \zeta_{i}}\right)\right\} .
$$

We now differentiate the condition $\partial S_{\omega} / \partial \omega=0$ with respect to $\zeta_{i}$. This yields

$$
\frac{\partial^{2} S_{\omega}}{\partial \zeta_{i} \partial \omega}+\frac{\partial^{2} S_{\omega}}{\partial \omega^{2}} \frac{\partial \omega}{\partial \zeta_{i}}=0
$$

Using this result to eliminate $\left(\partial \omega / \partial \zeta_{i}\right)$ in Eq. (7.11), we find, after a little algebra, that

$$
\frac{\partial^{2} S_{\mathrm{cl}}\left(\bar{\zeta}_{f}, \zeta_{i}, T\right)}{\partial \bar{\zeta}_{f} \partial \zeta_{i}}=\frac{2 j e^{-i \omega T}}{\left(1+\bar{\zeta}_{f} z(T)\right)^{2}} \cdot \frac{i T j^{2}}{2 \mu} \cdot\left(\frac{\partial^{2} S_{\omega}}{\partial \omega^{2}}\right)^{-1}
$$

Substituting Eqs. (7.5), (7.7), and (7.13) into the basic semiclassical form (4.20) for the propagator, we obtain 


$$
K_{\mathrm{scl}}=\sum_{\omega}\left(\frac{i T j^{2}}{2 \mu}\right)^{1 / 2}\left(\frac{\partial^{2} S_{\omega}}{\partial \omega^{2}}\right)^{-1 / 2} \exp \left\{S_{\omega}-\frac{i T}{2}(\omega+j \nu)+\frac{i}{2} \int_{0}^{T} A d t\right\} .
$$

The sum over $\omega$ is to be performed over all solutions to Eq. (7.6).

The utility of $S_{\omega}\left(\bar{\zeta}_{f}, \zeta_{i}, T\right)$ is not hard to understand. We are trying to evaluate

$$
\left\langle\zeta_{f}\left|e^{-i \nu \hat{J}_{3}^{2} T}\right| \zeta_{i}\right\rangle=\sum_{m=-j}^{m=j}\left(\bar{\zeta}_{f} \zeta_{i}\right)^{j+m} \frac{2 j !}{(j+m) !(j-m) !} e^{-i \nu m^{2} T},
$$

while we already know that

$$
\begin{aligned}
\left\langle\zeta_{f}\left|e^{-i \omega \hat{J}_{3} T}\right| \zeta_{i}\right\rangle & =\sum_{m=-j}^{m=j}\left(\bar{\zeta}_{f} \zeta_{i}\right)^{j+m} \frac{2 j !}{(j+m) !(j-m) !} e^{-i \omega m T} \\
& =\left(1+e^{-i \omega T} \bar{\zeta}_{f} \zeta_{i}\right)^{2 j} e^{i \omega j T} \\
& =\exp S_{\omega 0}\left(\bar{\zeta}_{f}, \zeta_{i}, T\right),
\end{aligned}
$$

where

$$
S_{\omega 0}\left(\bar{\zeta}_{f}, \zeta_{i}, \omega\right)=2 j \ln \left(1+e^{-i \omega T} \bar{\zeta}_{f} \zeta_{i}\right)+i T j \omega
$$

From the identity

$$
e^{-i \nu m^{2} T}=e^{-i \frac{\pi}{4}} \sqrt{\frac{T}{4 \pi \nu}} \int_{-\infty}^{\infty} d \omega e^{-i \omega m T} e^{i \omega^{2} T / 4 \nu}
$$

we have the exact relation

$$
\begin{aligned}
\left\langle\zeta_{f}\left|e^{-i \nu \hat{J}_{3}^{2} T}\right| \zeta_{i}\right\rangle & =e^{-i \frac{\pi}{4}} \sqrt{\frac{T}{4 \pi \nu}} \int d \omega\left\langle\zeta_{f}\left|e^{-i \omega \hat{J}_{3}}\right| \zeta_{i}\right\rangle e^{i \frac{\omega^{2} T}{4 \nu}} \\
& =e^{-i \frac{\pi}{4}} \sqrt{\frac{T}{4 \pi \nu}} \int d \omega \exp \left\{S_{\omega 0}\left(\bar{\zeta}_{f}, \zeta_{i}, \omega\right)+i \frac{\omega^{2} T}{4 \nu}\right\} \\
& =e^{-i \frac{\pi}{4}} \sqrt{\frac{T}{4 \pi \nu}} \int d \omega \exp \left\{2 j \ln \left(1+e^{-i \omega T} \bar{\zeta}_{f} \zeta_{i}\right)+i T\left\{j \omega+\frac{\omega^{2}}{4 \nu}\right\}\right\} .
\end{aligned}
$$

Given the form of the classical action (7.7), that $\mu \approx j^{2} \nu$, and the occurrence of $\left(\partial^{2} S_{\omega} / \partial \omega^{2}\right)^{-1 / 2}$ in the prefactor, it is clear that the semiclassical approximation is attempting a stationary phase approximation to this integral over $\omega$. That this approximation is indeed indicated can be seen by evaluating $\left(\partial^{2} S_{\omega} / \partial \omega^{2}\right)$. From Eqs. (7.9) and (7.6), we find

$$
\frac{\partial^{2} S_{\omega}}{\partial \omega^{2}}=\frac{i T j^{2}}{2 \mu}-\frac{1}{2} j T^{2}\left(1-\frac{j^{2} \omega^{2}}{4 \mu^{2}}\right)
$$


which scales as $j$ as $j \rightarrow \infty$.

We now write the exponent in Eq. (7.19) as $S_{\omega}-i T j \omega^{2} / 8 \mu$. Since the second term is $O\left(j^{0}\right)$ as $j \rightarrow \infty$, we may regard it as part of the pre-exponential factor in carrying out the stationary phase integral. In this way, we obtain

$$
K_{\text {exact }} \approx \sum_{\omega}\left(\frac{i T}{2 \nu}\right)^{1 / 2}\left(\frac{\partial^{2} S_{\omega}}{\partial \omega^{2}}\right)^{-1 / 2} \exp \left\{S_{\omega}-i T \frac{j \omega^{2}}{8 \mu}\right\} .
$$

The pre-exponential factors in the preceding equation agree with those in Eq. (7.14) to terms of order unity. To see whether the exponents agree, we must discuss the effect of the Solari-Kochetov phase. We find that

$$
\begin{aligned}
A & =\frac{1}{2}\left(\frac{\partial}{\partial \bar{z}} \frac{(1+\bar{z} z)^{2}}{2 j} \frac{\partial H}{\partial z}+\frac{\partial}{\partial z} \frac{(1+\bar{z} z)^{2}}{2 j} \frac{\partial H}{\partial \bar{z}}\right) \\
& =\omega+\frac{4 \mu}{j} \frac{\bar{z} z}{(1+\bar{z} z)^{2}} \\
& =\left(\omega+\frac{\mu}{j}\right)-\frac{j \omega^{2}}{4 \mu} .
\end{aligned}
$$

The term in parentheses serves to cancel [up to $O(1)$ ] the second term in the exponent in Eq. (7.14), and the $j \omega^{2} / 4 \mu$ term serves to correct $S_{\omega}$ as needed in Eq. (7.21). Thus our semiclassical formula is indeed accurate up to $O(1)$ as $j \rightarrow \infty$, and we may be confident that spectral properties (Kramers degeneracy in particular) derived from it by constructing, say, the Green's function or density of states, will be faithfully given.

Having demonstrated the formal equivalence of $K_{\text {scl }}$ and $K_{\text {exact }}$, we turn to the actual nature of the solution. Let us first rewrite the self-consistency condition (7.6) as

$$
2 i \tilde{\omega} \tau+\ln \left(\frac{1+\tilde{\omega}}{1-\tilde{\omega}}\right)=\ln \alpha
$$

where $\tilde{\omega}=j \omega / 2 \mu, \tau=\mu T / j$, and $\alpha=\bar{\zeta}_{f} \zeta_{i}$. In the limit $\tau \rightarrow \infty$, the left hand side of $(7.23)$ must remain finite, suggesting that $\tilde{\omega} \sim 1 / \tau$. A development in powers of $1 / \tau$ shows that we may write

$$
\tilde{\omega} \approx-\frac{i}{2} \frac{\ln \alpha}{\tau-i}-\frac{1}{24} \frac{(\ln \alpha)^{3}}{\tau^{4}}+O\left(\tau^{-5}\right)
$$


Since no restriction has been placed on which branch of $\ln \alpha$ is to be taken, this solution is infinitely multivalued, as asserted above. To leading order in $1 / \tau$, different solutions differ by additive amounts $n \pi / \tau$, where $n$ is an integer.

On the other hand, at $\tau=0$, Eq. (7.23) has a unique solution, $\tilde{\omega}=(\alpha-1) /(\alpha+1)$. The apparent contradiction with the earlier argument for an infinite number of solutions is resolved by noting that if, as $\tau \rightarrow 0$, we allow $\tilde{\omega}$ to diverge as $1 / \tau$, the left hand side of (7.23) again remains finite. Another development in powers of $\tau$ reveals that

$$
\tilde{\omega} \approx-\frac{i}{2} \frac{\ln (-\alpha)}{\tau}-\frac{2}{\ln (-\alpha)}-\frac{8 i}{[\ln (-\alpha)]^{3}} \tau+\cdots,
$$

which is also multivalued on account of the infinitely many branches of $\ln (-\alpha)$.

We can gain further insight into the nature of the propagator and the values of $\omega$ at the relevant stationary-phase points by working with initial and final states on the equator of the sphere: $\zeta_{i}=e^{i \phi_{i}}, \bar{\zeta}_{f}=e^{-i \phi_{f}}$. When $j$ is large, the problem should be essentially equivalent to a massive particle constrained to move on a ring of circumference $2 \pi$. If we write the hamiltonian for the latter as $L^{2} / 2 M$, where $L$ is the orbital angular momentum, and $M$ the mass, we expect the results for the two problems to be similar with $M=2 \nu$.

We start by considering the propagator for Larmor precession. Employing the leading large- $j$ estimate

$$
\frac{2 j !}{(j+m) !(j-m) !} \sim \frac{2^{2 j}}{\sqrt{\pi j}} e^{-m^{2} / j}
$$

and using the shorthand $\Delta \phi=\phi_{f}-\phi_{i}$, we may write

$$
\begin{aligned}
\left\langle\zeta_{f}\left|e^{-i \omega \hat{J}_{3}}\right| \zeta_{i}\right\rangle & =\sum_{m=-j}^{m=j}\left(\bar{\zeta}_{f} \zeta_{i}\right)^{j+m} \frac{2 j !}{(j+m) !(j-m) !} e^{-i \omega m T} \\
& \sim e^{-i j \Delta \phi} \frac{2^{2 j}}{\sqrt{\pi j}} \sum_{m} e^{-i m(\Delta \phi+\omega T)} e^{-m^{2} / j}
\end{aligned}
$$

If $T \gg j^{-1 / 2} / \omega$, the summand will have widely varying phases over the range of $m$ values that contributes to the sum, $|m| \sim \sqrt{j}$. By extending the sum over $m$ to infinity and using the Poisson summation formula (taking care that $m$ takes half-integer values when $j$ is half integral), we find 


$$
\left\langle\zeta_{f}\left|e^{-i \omega \hat{J}_{3}}\right| \zeta_{i}\right\rangle \approx e^{-i j \Delta \phi} 2^{2 j} \sum_{n} e^{-\frac{j}{4}(\Delta \phi+\omega T-2 \pi n)^{2}} \times(-1)^{n}
$$

where the $(-1)^{n}$ factor is present only when $j$ is half integral. This form is better suited to studying the large $j$ limit (for fixed $T$ ). In that case, (7.28), regarded as a function of $\omega$, is sharply peaked at $\omega=\bar{\omega}_{n}=(2 \pi n-\Delta \phi) / T$. These are the angular frequencies that allow uniform precession between $\phi_{i}$ and $\phi_{f}$ in time $T$. We now recall that Eq. (7.28) is nothing but $\exp \left(S_{\omega 0}\right)$. If we substitute this form into Eq. (7.19), and take into account the factor $\exp \left\{i \omega^{2} T / 4 \nu\right\}$ in determing the saddle-point frequencies, we find that they become complex

$$
\begin{aligned}
\omega_{n} & =\bar{\omega}_{n}\left(1-\frac{i}{\nu T j}\right)^{-1} \\
& \approx \bar{\omega}_{n}+\frac{i \bar{\omega}_{n}}{\nu T j} .
\end{aligned}
$$

Not surprisingly, this is just what we found in Eq. (7.24). The result reflects the fact that, to move at the required speed, the hamiltonian trajectories must move off the equator. There is then no real trajectory between the classical endpoints, and we must exploit the freedom to have trajectories where $\bar{\zeta}_{f} \neq z(T)^{*}$. When $j$ is large, however, Hamilton's equations provide large velocities close to the equator, and the imaginary parts of $\omega$ are correspondingly small. Performing the integration in Eq. (7.19), we find

$$
\left\langle e^{i \phi_{f}}\left|e^{-i \nu T J_{z}^{2}}\right| e^{i \phi_{i}}\right\rangle \approx 2^{2 j} e^{-i j \Delta \phi} \frac{1}{(1+i j \nu T)^{\frac{1}{2}}} \sum_{n} e^{-\frac{j}{4}(\Delta \phi-2 \pi n)^{2} /(1+i j \nu T)} \times(-1)^{n}
$$

where, again, the last factor is only present when $j$ is half-integral. This form should be compared with that for the massive particle 36]

$$
\left\langle\phi_{f}\left|e^{-i L^{2} T / 2 M}\right| \phi_{i}\right\rangle=\frac{1}{(2 \pi i M T)^{1 / 2}} \sum_{n} \exp \left(i n \Phi+i \frac{M(\Delta \phi-2 n \pi)^{2}}{2 T}\right) .
$$

We have incorporated an Aharonov-Bohm phase $\Phi$ into the result. This phase should be $\pi$ when we compare with half-integer spins, and the resulting pairwise degeneracy of the energy levels is the particle-on-a-ring analogue of Kramers degeneracy.

The similarity between Eqs. (7.30) and (7.31) is evident. Notice how $j$ sets the time scale for the crossover between the large- $T$ regime, where the spin behaves essentially as 
a particle of mass $2 \nu$ on the ring, and the short-time regime where the finite range of the coherent-state wavefunctions cuts off the $1 / \sqrt{T}$ divergence.

Note that we have ignored the difference between $\mu / j^{2}$ and $\nu$ in the above comparison, since as discussed while showing the equivalence of $K_{\mathrm{scl}}$ and $K_{\text {exact }}$, the error incurred is of order $1 / j^{2}$ relative to the leading term in the action. The semiclassical approximation therefore correctly obtains the first two terms in the large- $j$ expansion.

\section{DISCUSSION}

In the previous sections we have used the continuous-time path integral to motivate a semiclassical approximation to the coherent-state propagator for spin $j$. Although our derivation of the semiclassical propagator is purely formal, and the resulting expression must initially have only the status of a conjecture, we have demonstrated its correctness by verifying its short-time accuracy to $O\left(T^{2}\right)$, and checking its consistency under dissection of the path. From these two properties we may conclude that our expression is accurate to $O\left(j^{0}\right)$ uniformly in time.

In our derivation it was necessary to take into account an "anomaly" in the evaluation of the functional determinant of the Jacobi operator. This is the only place where we had to appeal to details of the discrete version of the path integral. Regulating the determinant in a manner consistent with the discrete path integral results in a correction to the naïve expression for the prefactor. This correction had been noted before, by Solari [24] and by Kochetov [25], but its importance does not seem to have been widely appreciated.

We have also discussed an example where an infinite number of classical trajectories contribute to the propagator. Here we again saw how the Solari-Kochetov factor is essential in obtaining the correct result.

A calculation of the Solari-Kochetov correction to the tunnel splitting between classically degenerate spin states will be reported in a separate publication. 


\section{ACKNOWLEDGMENTS}

Work at Urbana and Evanston was supported by the National Science Foundation under grants DMR-98-17941 and DMR-9616749, respectively. MS would also like to thank the TCM group at the Cavendish Laboratory, Cambridge, England, for hospitality, and the EPSRC for financial support under grant number GR/N00364.

\section{APPENDIX A: COMPOSITION OF PATH-DENSITY FACTORS}

In the this appendix we derive (6.12). We begin by restating the stationary phase conditions (6.5):

$$
\begin{aligned}
0 & =\frac{\partial S_{2}\left(\bar{\zeta}_{f}, \xi\right)}{\partial \xi}-\frac{2 j \bar{\xi}}{1+\bar{\xi} \xi} \\
0 & =\frac{\partial S_{1}\left(\bar{\xi}, \zeta_{i}\right)}{\partial \bar{\xi}}-\frac{2 j \xi}{1+\bar{\xi} \xi}
\end{aligned}
$$

Consider how the first of these evolves as we vary $\bar{\zeta}_{f}$. We find that

$$
\begin{aligned}
0 & =\frac{\partial}{\partial \bar{\zeta}_{f}}\left(\frac{\partial S_{2}}{\partial \xi_{c}}-\frac{2 j \bar{\xi}_{c}}{1+\bar{\xi}_{c} \xi_{c}}\right) \\
& =\frac{\partial^{2} S_{2}}{\partial \bar{\zeta}_{f} \partial \xi_{c}}+\frac{\partial^{2} S_{2}}{\partial \xi_{c}^{2}} \frac{\partial \xi_{c}}{\partial \bar{\zeta}_{f}}+\frac{2 j \bar{\xi}_{c}^{2}}{\left(1+\bar{\xi}_{c} \xi_{c}\right)^{2}} \frac{\partial \xi_{c}}{\partial \bar{\zeta}_{f}}-\frac{2 j}{\left(1+\bar{\xi}_{c} \xi_{c}\right)^{2}} \frac{\partial \bar{\xi}_{c}}{\partial \bar{\zeta}_{f}} \\
& =\frac{\partial^{2} S_{2}}{\partial \bar{\zeta}_{f} \partial \xi_{c}}+\frac{\partial \xi_{c}}{\partial \bar{\zeta}_{f}}\left(\frac{\partial^{2} S_{2}}{\partial \xi_{c}^{2}}+\frac{2 j \bar{\xi}_{c}^{2}}{\left(1+\bar{\xi}_{c} \xi_{c}\right)^{2}}\right)-\frac{2 j}{\left(1+\bar{\xi}_{c} \xi_{c}\right)^{2}} \frac{\partial \bar{\xi}_{c}}{\partial \bar{\zeta}_{f}}
\end{aligned}
$$

In the last line, we recognize the expression in parentheses to be $2 j \beta /\left(\left(1+\bar{\xi}_{c} \xi_{c}\right)^{2}\right.$, where $\beta$ is the coefficient appearing in (6.6). By differentiating each of the two stationary phase

conditions with respect to $\bar{\zeta}_{f}$ and $\zeta_{i}$, we get a total of four such equations. These may be summarized as

Taking determinants, we obtain

$$
\left(\begin{array}{cc}
1 & -\alpha \\
-\beta & 1
\end{array}\right)\left(\begin{array}{cc}
\frac{\partial \xi_{c}}{\partial \zeta_{i}} & \frac{\partial \xi_{c}}{\partial \bar{\zeta}_{f}} \\
\frac{\partial \bar{\xi}_{c}}{\partial \zeta_{i}} & \frac{\partial \bar{\xi}_{c}}{\partial \bar{\zeta}_{f}}
\end{array}\right)=\frac{\left(1+\bar{\xi}_{c} \xi_{c}\right)^{2}}{2 j}\left(\begin{array}{cc}
\frac{\partial^{2} S_{1}}{\partial \bar{\xi}_{c} \partial \zeta_{i}} & 0 \\
0 & \frac{\partial^{2} S_{2}}{\partial \bar{\zeta}_{f} \partial \xi_{c}}
\end{array}\right)
$$

$$
\left|\begin{array}{cc}
1 & -\alpha \\
-\beta & 1
\end{array}\right|\left|\begin{array}{cc}
\frac{\partial \xi_{c}}{\partial \zeta_{i}} & \frac{\partial \xi_{c}}{\partial \bar{\zeta}_{f}} \\
\frac{\partial \bar{\xi}_{c}}{\partial \zeta_{i}} & \frac{\partial \bar{\xi}_{c}}{\partial \bar{\zeta}_{f}}
\end{array}\right|=\frac{\left(1+\bar{\xi}_{c} \xi_{c}\right)^{4}}{(2 j)^{2}} \frac{\partial^{2} S_{1}}{\partial \bar{\xi}_{c} \partial \zeta_{i}} \frac{\partial^{2} S_{2}}{\partial \bar{\zeta}_{f} \partial \xi_{c}}
$$


We now recall that the gaussian integration in Eq. (6.9) leads to the inverse-square root of the precisely the first determinant in Eq. (A4). This equation expresses this determinant in terms of the second derivatives of $S_{1}$ and $S_{2}$, and the jacobian $\partial\left(\xi_{c}, \bar{\xi}_{c}\right) / \partial\left(\zeta_{i}, \bar{\zeta}_{f}\right)$. The derivatives of $S_{1}$ and $S_{2}$ will cancel with the prefactors in Eq. (6.9), leaving only the jacobian. We therefore turn to its evaluation, and show that it can be written in terms of the second derivatives of $S_{\text {tot }}$ with respect to $\bar{\zeta}_{f}$ and $\zeta_{i}$. We express $S_{\text {tot }}$ as

$$
S_{\text {tot }}=S_{2}+S_{1}-2 j \ln (1+\bar{\xi} \xi)
$$

and take note of the fact that both $\xi_{c}$ and $\bar{\xi}_{c}$ vary as we vary $\bar{\zeta}_{f}$ and $\zeta_{i}$. We have

$$
\begin{aligned}
\frac{\partial^{2} S_{\mathrm{tot}}}{\partial \bar{\zeta}_{f} \partial \zeta_{i}} & =\frac{\partial}{\partial \bar{\zeta}_{f}}\left(\frac{\partial S_{2}}{\partial \xi_{c}} \frac{\partial \xi_{c}}{\partial \zeta_{i}}-\frac{2 j \bar{\xi}_{c}}{1+\bar{\xi} \xi} \frac{\partial \xi_{c}}{\partial \zeta_{i}}+\frac{\partial S_{1}}{\partial \bar{\xi}_{c}} \frac{\partial \bar{\xi}_{c}}{\partial \zeta_{i}}+\frac{\partial S_{1}}{\partial \zeta_{i}}-\frac{2 j \xi_{c}}{1+\bar{\xi} \xi} \frac{\partial \bar{\xi}_{c}}{\partial \zeta_{i}}\right) \\
& =\frac{\partial}{\partial \bar{\zeta}_{f}}\left(\frac{\partial \xi_{c}}{\partial \zeta_{i}}\left\{\frac{\partial S_{2}}{\partial \xi_{c}}-\frac{2 j \bar{\xi}_{c}}{1+\bar{\xi} \xi}\right\}+\left(\frac{\partial S_{1}}{\partial \zeta_{i}}\right)_{\bar{\xi}_{c}}+\left\{\frac{\partial S_{1}}{\partial \bar{\xi}_{c}}-\frac{2 j \xi_{c}}{1+\bar{\xi} \xi}\right\} \frac{\partial \bar{\xi}_{c}}{\partial \zeta_{i}}\right) .
\end{aligned}
$$

The expressions in braces in the last line are the stationary phase conditions, so they are zero, as are their derivatives. Thus:

$$
\frac{\partial^{2} S_{\mathrm{tot}}}{\partial \bar{\zeta}_{f} \partial \zeta_{i}}=\frac{\partial}{\partial \bar{\zeta}_{f}}\left(\frac{\partial S_{1}}{\partial \zeta_{i}}\right)_{\bar{\xi}_{c}}=\frac{\partial^{2} S_{1}}{\partial \bar{\xi}_{c} \partial \zeta_{i}} \frac{\partial \bar{\xi}_{c}}{\partial \bar{\zeta}_{f}} .
$$

Taking note of the fact that the derivative of $S_{1}$ with respect to $\zeta_{i}$ is at fixed $\bar{\xi}_{c}$, while we have useful expressions for the derivative including the variation of $\bar{\xi}_{c}$, we interchange the order of differentiation, and write

$$
\begin{aligned}
\frac{\partial^{2} S_{\mathrm{tot}}}{\partial \bar{\zeta}_{f} \partial \zeta_{i}} & =\frac{\partial \bar{\xi}_{c}}{\partial \bar{\zeta}_{f}}\left(\frac{\partial}{\partial \zeta_{i}}\left(\frac{\partial S_{1}}{\partial \bar{\xi}_{c}}\right)-\frac{\partial^{2} S_{1}}{\partial \bar{\xi}_{c}^{2}} \frac{\partial \bar{\xi}_{c}}{\partial \zeta_{i}}\right) \\
& =\frac{\partial \bar{\xi}_{c}}{\partial \bar{\zeta}_{f}} \frac{\partial}{\partial \zeta_{i}}\left(\frac{2 j \xi_{c}}{1+\bar{\xi}_{c} \xi_{c}}\right)-\frac{\partial \bar{\xi}_{c}}{\partial \bar{\zeta}_{f}} \frac{\partial^{2} S_{1}}{\partial \bar{\xi}_{c}^{2}} \frac{\partial \bar{\xi}_{c}}{\partial \zeta_{i}} \\
& =\frac{\partial \bar{\xi}_{c}}{\partial \bar{\zeta}_{f}} \frac{\partial}{\partial \zeta_{i}}\left(\frac{2 j \xi_{c}}{1+\bar{\xi}_{c} \xi_{c}}\right)-\frac{\partial \bar{\xi}_{c}}{\partial \zeta_{i}} \frac{\partial}{\partial \bar{\zeta}_{f}}\left(\frac{2 j \xi_{c}}{1+\bar{\xi}_{c} \xi_{c}}\right) \\
& =\frac{2 j}{\left(1+\bar{\xi}_{c} \xi_{c}\right)^{2}}\left(\frac{\partial \bar{\xi}_{c}}{\partial \bar{\zeta}_{f}} \frac{\partial \xi_{c}}{\partial \zeta_{i}}-\frac{\partial \bar{\xi}_{c}}{\partial \zeta_{i}} \frac{\partial \xi_{c}}{\partial \bar{\zeta}_{f}}\right)
\end{aligned}
$$

In going from the second line to the third, we used one of the equations from (A3).

Putting this together with (A4) yields 


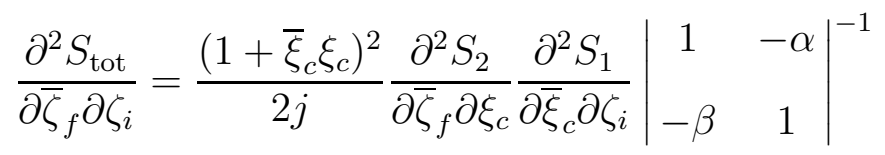

which is identical to Eq. (6.12) 


\section{REFERENCES}

[1] J. R. Klauder, Phys. Rev. D 19 (1979) 2349.

[2] H. Kuratsuji, T. Suzuki, J. Math. Phys. 21 (1980) 472.

[3] A. Jevicki, N. Papanicolaou, Ann. Phys. (N.Y.) 120 (1979) 107.

[4] H. B. Nielsen, D. Röhrlich, Nucl. Phys. B299 (1988) 471.

[5] J. R. Klauder, in Path Integrals Proceedings of the NATO Advanced Summer Institute, edited by G. J. Papadopoulos and J. T. Devreese (Plenum, NY ,1978).

[6] Path integrals and coherent states of $S U(2)$ and $S U(1,1)$, H. Kuratsuji, A. Inomata, C. C. Gerry, eds. (World Scientific, Singapore, 1992).

[7] N. M. J. Woodhouse, Geometric Quantization (Oxford University Press 1992).

[8] J. H. Duistermat, G. J. Heckman, Invt. Math. 69 (1982) 259; 72(1983) 153.

[9] M. Stone, Nucl. Phys. B 314 (1989) 577.

[10] E. Keskivakkuri, A. J. Niemi, G. Semenoff, O. Tirkkonen, Physical Review D 44 (1991) 3899.

[11] E. M. Chudnovsky, L. Gunther, Phys. Rev. Lett. 60 (1988) 661.

[12] D. Loss, D. P. Vincenzo, G. Grinstein, Phys. Rev. Lett. 69 (1992) 3232.

[13] J. von Delft and C. L. Henley, Phys. Rev. Lett. 69 (1992) 3236.

[14] A. Garg, Europhys. Lett. 22, 205 (1993).

[15] W. Wernsdorfer and R. Sessoli, Science 284, 133 (1999).

[16] H. Kuratsuji, Y. Mizobuchi, J. Math. Phys. 22 (1981) 757.

[17] A. Garg, G-H. Kim, Phys. Rev. B 45 (1992) 921.

[18] M. Enz, R. Schilling, J. Phys. C 19 (1986) L711; 1765. 
[19] V. I. Belinicher, C. Providencia, J. da Providencia, J. Phys. A 30 (1997) 5633.

[20] J. Shibata, S. Takagi, Int. Jour. Mod. Phys. B 13 (1999) 107.

[21] J. L Hemmen A. Sütő, Europhys. Lett. 1 (1986) 481; Physica B \& C 141 (1986) 37.

[22] A. Garg, Phys. Rev. Lett. 83, 4385 (1999); cond-mat/0003114; cond-mat/0003156.

[23] J. Villain and A. Fort, submitted to Europhys. B.

[24] H. G. Solari, J. Math. Phys. 27 (1987) 1097.

[25] E. A. Kochetov, J. Math. Phys. 36 (1995) 4667.

[26] A. Perelomov, Generalized Coherent States and their Applications, (Springer-Verlag, Berlin 1986).

[27] See for example: R. Bott, Ann. Math. 66 (1957) 203.

[28] A. A. Kirillov, Elements of the Theory of Representations, (Springer-Verlag, Berlin, New-York, 1976).

[29] E. Lieb, Comm. Math. Phys. 31 (1973) 327.

[30] See for example: R. D. Richtmyer, Principles of Advanced Mathematical Physics, (Springer Verlag, Berlin, New-York, 1978), section 7.2.

[31] A. P. Balachandran, G. Marmo, B. S. Skagerstam, A. Stern, Gauge Symmetries and Fibre Bundles, (Springer-Verlag, Berlin, 1983); A. P. Balachandran, G. Marmo, B. S. Skagerstam, A. Stern, Classical topology and Quantum States, (Springer-Verlag, Berlin, 1991).

[32] The "shooting method" we used is described in: S. Kos, M. Stone, Phys. Rev. B 59 (1999) 9545. (cond-mat/9809182)

[33] M. S. Marinov, Phys. Rep. 60 (1980) 1. 
[34] E. Ercolessi, G. Morandi, F. Napoli, R. Pieri, J. Math. Phys. 37 (1996) 535.

[35] F. Funahashi, T. Kashiwa, S. Sakoda, K. Fujii, J. Math. Phys. 36 (1995) 3232.

[36] See, e.g., L. S. Schulman, Techniques and Applications of Path Integration, (Wiley, New York, 1981), Chapter. 23. 